\title{
Unlimited Niche Packing in a Lotka-Volterra Competition Game
}

\author{
Ross Cressman \\ Department of Mathematics \\ Wilfrid Laurier University \\ Waterloo, ON N2L 3C5 Canada \\ rcressman@wlu.ca \\ Abdel Halloway \\ Department of Biological Sciences \\ University of Illinois at Chicago \\ Chicago, IL 60607 USA \\ ahallo2@uic.edu \\ Gordon G. McNickle \\ Biology Department \\ Wilfrid Laurier University \\ Waterloo, ON N2L 3C5 Canada \\ gmcnickle@wlu.ca \\ Joe Apaloo \\ Department of Mathematics, Statistics and Computer Science \\ St. Francis Xavier University \\ P. O. Box 5000 \\ Antigonish, Nova Scotia B2G 2W5 Canada \\ japaloo@stfx.ca \\ Joel S. Brown
}


Department of Biological Sciences

University of Illinois at Chicago

Chicago, IL 60607 USA

squirrel@uic.edu

\section{Thomas L. Vincent}

Department of Aerospace and Mechanical Engineering

University of Arizona

Tucson, AZ 85721 USA

Keywords: ESS, convergence stable, NIS, Nash equilibrium, infinite niche packing, Darwinian dynamics.

\section{Abstract}

A central question in the study of ecology and evolution is: "Why are there so many species?" It has been shown that certain forms of the Lotka-Volterra (L-V) competition equations lead to an unlimited number of species. Furthermore, these authors note how any change in the nature of competition (the competition kernel) leads to a finite or small number of coexisting species. Here we build upon these works by further investigating the L-V model of unlimited niche packing as a reference model and evolutionary game for understanding the environmental factors restricting biodiversity. We also examine the combined eco-evolutionary dynamics leading up to the species diversity and traits of the ESS community in both infinite and finite niche-packing versions of the model. As an L-V game with symmetric competition, we let the strategies of individuals determine the strength of the competitive interaction (like competes most with like) and also the carrying capacity of the population. We use a mixture of analytic proofs (for one and two species systems) and numerical simulations. For the model 
of unlimited niche packing, we show that a finite number of species will evolve to specific convergent stable minima of the adaptive landscape (also known as species archetypes). Starting with a single species, faunal buildup can proceed either through species doubling as each diversity-specific set of minima are reached, or through the addition of species one-by-one by randomly assigning a speciation event to one of the species. Either way it is possible for an unlimited number or species to evolve and coexist. We examine two simple and biologically likely ways for breaking the infinite niche-packing: 1) some minimum level of competition among species, and 2) constrain the fundamental niche of the trait space to a finite interval. When examined under both ecological and evolutionary dynamics, both modifications result in convergent stable ESSs with a finite number of species. When the number of species is held below the number of species in an ESS coalition, we see a diverse array of convergent stable niche archetypes that consist of some species at maxima and some at minima of the adaptive landscape. Our results support those of others and suggest that instead of focusing on why there are so many species we might just as usefully ask, why are there so few species? 


\section{Introduction}

Hutchinson (1959) in his "Homage to Santa Rosalia" asks "Why are there so many species?" This succinctly summed up and anticipated the enduring and important question of what promotes and maintains biodiversity. The development of the Lotka-Volterra (L-V) competition equations (Volterra 1926, 1928, Lotka 1927) and Gause's (1931, 2003) empirical application of this model led to Gause's Principle or the Competitive Exclusion Principle. For similar species to coexist they must occupy separate niches. With explicit resource dynamics in models of competition, this general result requires that the number of coexisting species cannot exceed the number of distinct resources (including both opportunities and hazards) (Tilman 1982). In typical consumer-resource models the niche axis offers discrete resources such as A or B with nothing in between.

What happens when the niche axis is continuous? Does this provide an infinite number of resources and hence the potential for unlimited niche packing? MacArthur (1958) found five species of warblers dividing up the foliage height and breadth of conifers - five species and five foliage habitats. Yet, in reality the number of habitats recognized by MacArthur was a continuum. The presence of the five bird species in somewhat discrete and predictable locations within the tree defined the five habitats. Fewer or more species would have led to different conclusions. A finite number of distinct species coexisting on continuous niche axes has led to an interest in limiting similarity and Hutchinson's ratios (MacArthur and Levins 1967). Key questions that are central to the study of ecology but for which we still do not have clear answers are: How similar can two species be to each other and still coexist? Do traits such as the ratio of body sizes, beak sizes, or other functional traits associated with close competitors provide useful insights into community organization and species coexistence?

Just as the Lotka-Volterra competition equations provided useful insights into the coexis- 
tence or exclusion of species from a community, they have provided an equally valuable tool for modelling and understanding eco-evolutionary dynamics (Morris and Lundberg 2011) where the number of species and their traits are allowed to evolve in response to ecological interactions. Roughgarden (1979) used Gaussian functions for the resource axis (distribution of carrying capacities) and for the competition coefficients (like competes most with like) to show how unlimited niche packing can occur along any discrete interval of the resource axis. In essence, the community of competitors becomes a continuum of species whose share in the community also follows a Gaussian curve (Roughgarden 1979, Barabás et al. 2012).

A number of empirical objections can be raised regarding this model of unlimited niche packing: sexual reproduction, minimum viable population sizes, boundaries to the length of resource axes, deviations from the idealized Gaussian distributions just to name a few (Gyllenberg and Meszéna 2005, Barabás et al. 2012). Indeed, modifications of the L-V model as an evolutionary game have provided insights into niche coevolution, speciation, and community structure where the number of species at the eco-evolutionary equilibrium involve a fixed number of species with discrete traits (Brown and Vincent 1987, 1992, Metz et al. 1996, Cohen et al. 1999, Ripa et al 2009). Here we build upon and extend these prior works. We add to prior work of the L-V model with unlimited niche packing by examining the ecological and evolutionary dynamics that can lead to faunal buildup. This model provides an idealized model that, while unlikely in nature, can be used as a starting point for understanding how biodiversity in nature evolves and coexists. With a world full of resource continuum, we can rephrase Hutchinson's (1959) observation of "why are there so many species?" to "why are there so few species?". Unlimited niche packing is possible in theory, and yet seem in nature. Asking why not might provide new insights into questions of biodiversity. The L-V competition evolutionary game with infinite niche packing provides an excellent reference model for understanding limiting similarity, Hutchinson's ratios, and community organization. It also complements other game theory models of 
species coexistence based on predator-prey models (Ripa et al. 2009) such as the cyclic dynamics seen in versions of rock-paper-scissor games (Szolnoki et al. 2014).

Our goal is to explore Roughgarden's model of infinite niche packing in greater detail that explicitly considers evolutionary dynamics, and the properties of various convergent stable points of the adaptive landscape when species numbers fall below that of the ESS. We will do this as an evolutionary game while preserving the resource and competition curves proposed by Roughgarden (1979) and studied extensively by others (Bulmer 1974 ; Sasaki and Ellner 1995; Sasaki 1997; Szabó and Meszéna 2006; Parvinen and Meszéna 2009; Barabás et al. 2013; D'Andrea et al. 2013). The model produces unlimited niche packing and a continuum of species with specfic characteristics and population sizes (Meszéna et al. 2006;Barabás and Meszéna 2009). What emerges is a model of faunal buildup (taken as far as 1024 coexisting species) through continual adaptive speciation as the number of species grows towards unlimited niche packing. In this model, we show that any finite number of species will evolve to a convergent stable, non-ESS, "niche archetype". While mostly demonstrated by simulations we obtain analytic solutions for the two species case which allows us draw explicit conclusions regarding the effects of niche breadth and competition parameters on character divergence and population sizes. In going towards an unlimited number of species, we show that the ultimate contiguous distribution of "species" is a gametheoretic, Nash solution. We show that the convergent stable community of 1024 species essentially fits this distribution. We then follow the lead of those (Gyllenberg and Meszéna 2005;Barabás et al. 2012) who shown that the competition functions that permit unlimited niche packing are not robust. Any modification to the functional form leads to a collapse of the continuum of coexisting species to finite numbers. Here, we add eco-evolutionary dynamics to two modifications of the competition function to study the diversity of species at the ESS, and faunal buildup to the ESS startling with a one or a reduced number of species Finally, we add eco-evolutionary dynamics to a modified competition function proposed by 
Leimer et al. (2013) characterized by the possibility of unlimited niche packing within the context of convergent stable maxima rather than minima. Ultimately, the factors that cause the continuum of species to cease may be the model's greatest value for understanding what actually happens in the nitty-gritty of real biological communities in nature.

\section{An Eco-Evolutionary Lotka-Volterra Model}

Following Roughgarden (1979) and Brown and Vincent (1987), we develop an evolutionary game based on the Lotka-Volterra competition model. To do this we imagine a continuous valued trait, $u$, that can potentially take on any value from negative infinity to positive infinity. Carrying capacity, $K$, is influenced by the individual's own strategy, $v$. We let the vector $\mathbf{u}=\left(u_{1}, \ldots, u_{n}\right)$ denote the different strategies currently found among individuals within the ecological community. For this paper, we will assume that the different $u_{i}$ 's represent distinct strategies associated with different species. Furthermore, we assume that species breed true. The total number of species currently within the community is $n$. The vector $\mathbf{x}=\left(x_{1}, \ldots, x_{n}\right)$ represents the current population sizes of each of the species such that $x_{i}$ is the population size of species $u_{i}$. With these assumptions, we can now write the expected per capita growth rate (i.e. fitness) of a focal individual using strategy, $v$, as a Lotka-Volterra competition game:

$$
G(v, \mathbf{u}, \mathbf{x})=\frac{r}{K(v)}\left[K(v)-\sum_{j=1}^{n} a\left(v, u_{j}\right) x_{j}\right]
$$

We assume that competition is most intense between individuals using the same strategy and that competition between two species declines as the difference in their strategy values increases. Like competes most with like. Hence, we let the competition coefficient, $a\left(v, u_{j}\right)$, be a Gaussian relationship based on the difference between the focal individual's strategy 
and that of the competitor. The function reaches a maximum of 1 when $v=u_{i}$ and declines as the strategies of the competitors diverge (figure $1 \mathrm{~A}$ ). This yields the following:

$$
a\left(v, u_{i}\right)=\exp \left[-\frac{\left(v-u_{i}\right)^{2}}{2 \sigma_{a}^{2}}\right]
$$

For the carrying capacity we assume a Gaussian relationship between the individual's strategy, $v$, and $K$. The relationship is centered on $v=0$ such that $K(v)$ reaches a maximum of $K_{m}$ when $v=0$ and then $K(v)$ declines as $v$ deviates from 0 (figure $1 \mathrm{~B}$ ). This yields the following:

$$
K(v)=K_{m} \exp \left[-\frac{v^{2}}{2 \sigma_{k}^{2}}\right]
$$

Two parameters will be key to the properties and dynamics of this model. These are the width of the niche axis, $\sigma_{k}^{2}$, and the width of the competition coefficient, $\sigma_{a}^{2}$. As $\sigma_{k}^{2}$ increases, the width of the niche axis increases and the decline in $K(v)$ as the individual's strategy deviates from $v=0$ becomes less severe (figure $1 \mathrm{~B}$ ). As $\sigma_{a}^{2}$ increases, an individual receives less of a benefit through a reduction in competition by deviating its strategy from the strategies of others. With a large $\sigma_{a}^{2}$, the competition coefficient, $a\left(v, u_{i}\right)$, declines slowly with $\left|v-u_{i}\right|$ (figure $1 \mathrm{~A}$ ).

This model has been analyzed using Darwinian Dynamics (Vincent et al. 1993, Vincent and Brown 2005). The Gfunction can be used to determine both population dynamics, $\frac{\partial x_{i}}{\partial t}$, and evolutionary dynamics, $\frac{\partial u_{i}}{\partial t}$, as follows:

$$
\frac{\partial x_{i}}{\partial t}=x_{i} G(v, \mathbf{u}, \mathbf{x}) \text { when } v=u_{i} \quad i=1, \cdots, n \quad \text { (ecological dynamics) }
$$

and 


$$
\frac{\partial u_{i}}{\partial t}=k \frac{\partial G}{\partial v} \text { when } v=u_{i} \quad i=1, \cdots, n \quad \text { (evolutionary dynamics) }
$$

where the positive parameter $k$ represents some measure of additive genetic variance and heritability of the trait. These eco-evolutionary dynamics conform to Fisher's Fundamental Theorem of Natural Selection (Fisher 1958) and models of Adaptive Dynamics (Metz et al. 1996, Gertiz et al. 1998). The outcome of these systems (i.e. the long-run behavior of their solution trajectories) is often analyzed through the concepts of evolutionarily stable strategy (ESS) and convergence stability.

The definitions for ESS and convergence stability are not universal. In some cases the ESS has been defined as a strategy that maximizes the fitness function (see e.g., Leimar 2009) and in some cases as an asymptotically stable equilibrium point of an evolutionary dynamic (see e.g., Vincent and Brown 2005). These definitions apply to both single species and multi species evolutionary games. For single species, convergence stability is well defined as a strategy that is approached through strategy substitution (Eshel and Motro 1981, Christiansen 1991). However, for multi species evolution, there is no clear definition for convergence stability. Cressman (2009) has provided a definition for two species evolution and Leimar (2009) has discussed convergence stability for nspecies models. In this manuscript, we adopt the following operationalizable definitions.

Definition 1 An ESS is an eco-evolutionary equilibrium $\left(\mathbf{u}^{*}, \mathbf{x}^{*}\right)$ with a finite number $n$ of strategy types $u_{1}^{*}, u_{2}^{*}, \ldots, u_{n}^{*}$, and positive $x_{1}^{*}, x_{2}^{*}, \ldots, x_{n}^{*}$ such that $G\left(u_{i}^{*} ; \mathbf{u}^{*}, \mathbf{x}^{*}\right)=0$ for $i=1, \ldots, n$ and $G\left(v ; \mathbf{u}^{*}, \mathbf{x}^{*}\right)<0$ if $v$ does not equal any $u_{i}^{*}$.

The above definition of ESS is essentially the ESS Maximum Principle (Vincent and Brown 2005). Thus ESS are strategies which reside at (global) peaks of the adaptive landscape with peak value of $G(\cdot)=0$. 
Definition 2 A convergence stable strategy is an eco-evolutionary equilibrium $\left(\mathbf{u}^{*}, \mathbf{x}^{*}\right)$ with a finite number $n$ of strategy types $u_{1}^{*}, u_{2}^{*}, \ldots, u_{n}^{*}$, and positive $x_{1}^{*}, x_{2}^{*}, \ldots, x_{n}^{*}$ such that $\left(\mathbf{u}^{*}, \mathbf{x}^{*}\right)$ is an asymptotically stable equilibrium for the 2ndimensional eco-evolutionary dynamics (4) and (5).

Thus, a convergence stable strategy is taken operationally to be a strategy (or niche archetype) that are rest points of the eco-evolutionary dynamics (4) and (5) which may be at a maximum, minimum or inflection point of the fitness landscape with fitness of the extant strategies being 0. This evolutionary stability idea is similar to that in Leimar (2009) except that he does not consider population sizes explicitly.

Vincent and Brown (2005) define niche archetypes as any community of $n$ species' strategies that converge on a stable $\mathbf{u}^{*}$ and $\mathbf{x}^{*}$. They represent communities that result from the ecological and evolutionary dynamics. Such communities may not be ESS. Some or all of the species may reside at local peaks, minima, or even inflection points of the adaptive landscape. But in the absence of additional species, such communities would remain persistent and stable to some range of perturbations to the existing species' strategy values or population sizes.

Since fitness is defined here as per capita population growth rate, at an equilibrium population size $\mathbf{x}^{*}$, where all of the $n$ species have positive population sizes, each species fitness must equal 0 . This requires that $G\left(v, \mathbf{u}, \mathbf{x}^{*}\right)=0$ when $v=u_{i}$ for all $i=1, \cdots, n$ :

$$
K(v)=\sum_{j=1}^{n} a\left(v, u_{j}\right) x_{j}^{*} \text { when } v=u_{i} \quad i=1, \cdots, n
$$

Similarly, at an equilibrium of the evolutionary dynamics, the rate of change in each species' strategy must be 0 . This requires that $\frac{\partial G}{\partial v}=0$ when $v=u_{i}^{*}$ for all $i=1, \cdots, n$ : 


$$
\frac{\partial K(v)}{\partial v} \sum_{j=1}^{n} a\left(v, u_{j}\right) x_{j}=K(v) \sum_{j=1}^{n} \frac{\partial a}{\partial v} x_{j} \text { when } v=u_{i}^{*} \quad i=1, \cdots, n
$$

At a combined eco-evolutionary (i.e. Darwinian) equilibrium $\left(\mathbf{u}^{*}, \mathbf{x}^{*}\right)$, we can further simplify the ecological equilibrium conditions by substituting in the conditions for $x^{*}$ to obtain:

$$
\frac{\partial K(v)}{\partial v}=\sum_{j=1}^{n} \frac{\partial a\left(v, u_{j}\right)}{\partial v} x_{j}^{*} \text { when } v=u_{i}^{*} \quad i=1, \cdots, n
$$

While the properties of this model for $n=1$ are well understood (Apaloo 1997, 2003), it is nevertheless useful to show and catalog them as the basis for understanding the model for multiple coexisting species, $n>1$.

When there is just a single species, the equilibrium conditions result in $u^{*}=0$ and $x^{*}=K_{m}$ (figure 2). Applying the conditions of Apaloo et al. (2009), this solution is both convergent stable and a Neighborhood Invader Strategy (NIS). This means that this equilibrium point will result from the Darwinian dynamics (convergent stable) and furthermore, the strategy $u^{*}=0$ can successfully invade (i.e. increase when rare) populations where all individuals in the population are using a strategy $u \neq 0$ at their ecological equilibrium of $x^{*}(u)=K(u)<K_{m}$. The eco-evolutionary solution of $u^{*}=0$ and $x^{*}=K_{m}$ makes sense. This strategy is the one that maximizes carrying capacity and results in the largest equilibrium population size when there is just one species.

Is this solution an ESS in the sense of Definition 1 (i.e. maximizing fitness given the circumstances)? This solution would be an ESS if it resides on the peak of the adaptive landscape $\left(\frac{\partial^{2} G}{\partial v^{2}}<0\right.$, figure $\left.2 \mathrm{~A}-\mathrm{C}\right)$ and an evolutionarily stable minimum (sensu Abrams et al. 1993) if it resides at a minimum of the adaptive landscape $\left(\frac{\partial^{2} G}{\partial v^{2}}>0\right.$, figure $2 \mathrm{D}$ F). For a single species, the relationship between the breadth of the niche axis and the 
breadth of competitive interactions determines whether $u^{*}=0$ is an $\operatorname{ESS}\left(\sigma_{k}^{2}<\sigma_{a}^{2}\right)$ or a convergent stable minimum $\left(\sigma_{k}^{2}>\sigma_{a}^{2}\right)$ (figure 2). For a single species at $u^{*}=0$, the carrying capacity function produces stabilizing selection for maximum $K$. Conversely, the competition function produces disruptive selection because like competes most with like. With a narrow niche axis and broad competitive interactions, the stabilizing selection on $K$ outweighs the disruptive selection on $a$ and $u^{*}=0$ is a convergent stable ESS (figure 2 A-C). With a broad niche axis and narrow competitive interactions, the disruptive selection on $a$ outweighs the stabilizing selection $K$ and $u^{*}=0$ is a convergent stable minimum (figure 2 D-F).

For the remainder of this paper, we shall be interested in the case where $\sigma_{k}^{2}>\sigma_{a}^{2}$ and $u^{*}=0$ is a convergent stable minimum. Such minima have been identified as bifurcation points (Brown and Pavlovic 1992) that can produce evolutionary branching (Geritz at al. 1998) via adaptive speciation (Cohen et al. 1999, Doebeli and Dieckmann 2003; Ripa et al. 2009). In this case, we expect the single species at $u^{*}=0$ to split into two with different values of $u$ which diverge from $u^{*}=0$ as the difference $\sigma_{k}^{2}-\sigma_{a}^{2}$ increases. What happens when we have $n=2$ ?

\section{The Lotka-Volterra Competition Game with Two Species}

Under adaptive speciation at $u^{*}=0$, the single species at its ecological equilibrium $x^{*}=K_{m}$ splits into two species, whose strategies are initially close by and branch on either side of 0 (e.g., Geritz et al. 1998). The intense inter-specific competition between the two new species exerts selection for further evolutionary divergence. But, how far will they diverge, and will the two species continue to coexist at positive population sizes? Theoretically, several longrun possibilities exist for this speciation event, including one of the species going extinct and the surviving species evolving back to $u=0$ before another cycle of adaptive speciation 
occurs. Another possibility has the two species coexisting in separate niches, as they evolve to new points of evolutionary and ecological stability - such points would balance the benefits of further divergence with the cost of lowered carrying capacity. From extensive simulations of the Darwinian dynamics for $n=2$ (figure 3), the outcome of the $\mathrm{L}-\mathrm{V}$ competition model is the second scenario. A convergent stable equilibrium with two species placed symmetrically about $u=0$ always emerges. As expected, the two species have equal population sizes at equilibrium: $x_{1}^{*}=x_{2}^{*}$.

We can predict, a priori, what this new equilibrium will be since the system must then evolve to a combined eco-evolutionary equilibrium $\left(\mathbf{u}^{*}, \mathbf{x}^{*}\right)=\left(\left(u_{1}^{*}, u_{2}^{*}\right),\left(x_{1}^{*}, x_{2}^{*}\right)\right)$. That is, $u_{1}^{*} \neq u_{2}^{*}, x_{1}^{*}>0, x_{2}^{*}>0$ must satisfy:

$$
K(v)=\sum_{j=1}^{2} a\left(v, u_{j}\right) x_{j}^{*} \text { and } \frac{\partial K(v)}{\partial v}=\sum_{j=1}^{2} \frac{\partial a\left(v, u_{j}\right)}{\partial v} x_{j}^{*} \text { when } v=u_{i}^{*} \quad i=1,2
$$

This equilibrium is a solution to the following system of four equations in the four unknowns $u_{1}^{*}, u_{2}^{*}, x_{1}^{*}, x_{2}^{*}$.

$$
\begin{aligned}
x_{1}+x_{2} e^{-\left(u_{1}-u_{2}\right)^{2} / 2 \sigma_{a}^{2}} & =K_{m} e^{-u_{1}^{2} / 2 \sigma_{k}^{2}} \\
x_{1} e^{-\left(u_{1}-u_{2}\right)^{2} / 2 \sigma_{a}^{2}}+x_{2} & =K_{m} e^{-u_{2}^{2} / 2 \sigma_{k}^{2}} \\
\frac{u_{1}-u_{2}}{\sigma_{a}^{2}} e^{-\left(u_{1}-u_{2}\right)^{2} / 2 \sigma_{a}^{2}} x_{2} & =K_{m} \frac{u_{1}}{\sigma_{k}^{2}} e^{-u_{1}^{2} / 2 \sigma_{k}^{2}} \\
\frac{u_{2}-u_{1}}{\sigma_{a}^{2}} e^{-\left(u_{1}-u_{2}\right)^{2} / 2 \sigma_{a}^{2}} x_{1} & =K_{m} \frac{u_{2}}{\sigma_{k}^{2}} e^{-u_{2}^{2} / 2 \sigma_{k}^{2}} .
\end{aligned}
$$

If the equilibrium is symmetric about 0 with $u_{1}^{*}=u^{*}>0$ and $u_{2}^{*}=-u_{1}^{*}$, then the first two equations imply that $x_{1}^{*}=x_{2}^{*}=x^{*}$. The four equations reduce to two, 


$$
\begin{aligned}
x^{*} e^{-\left(2 u^{*}\right)^{2} / 2 \sigma_{a}^{2}}+x^{*} & =K_{m} e^{-u^{* 2} / 2 \sigma_{k}^{2}} \\
\frac{2 u^{*}}{\sigma_{a}^{2}} e^{-\left(2 u^{*}\right)^{2} / 2 \sigma_{a}^{2}} x^{*} & =K_{m} \frac{u^{*}}{\sigma_{k}^{2}} e^{-u^{* 2} / 2 \sigma_{k}^{2}},
\end{aligned}
$$

which can be solved (see Appendix A), yielding the unique symmetric equilibrium

$$
\begin{aligned}
u^{* 2} & =\frac{\sigma_{a}^{2}}{2} \ln \left(\frac{2 \sigma_{k}^{2}-\sigma_{a}^{2}}{\sigma_{a}^{2}}\right) \\
x^{*} & =K_{m} \frac{\sigma_{a}^{2}}{2 \sigma_{k}^{2}}\left(\frac{2 \sigma_{k}^{2}-\sigma_{a}^{2}}{\sigma_{a}^{2}}\right)^{1-\frac{\sigma_{a}^{2}}{4 \sigma_{k}^{2}}} .
\end{aligned}
$$

There is something aesthetically pleasing about the effects of niche width, $\sigma_{k}^{2}$, and the width of the competition coefficient, $\sigma_{a}^{2}$, on the two species' strategy values and population sizes. Recall that for there to be a two species convergent stable point: $\sigma_{k}^{2}>\sigma_{a}^{2}$. First, notice what happens at the point where $\sigma_{k}^{2}=\sigma_{a}^{2}$. In this case, $u_{1}^{*}=u_{2}^{*}=u^{*}=0$ and both species would want the same strategy. Furthermore, each species would have the same $x^{*}=\frac{K_{m}}{2}$ for a total population size of $K_{m}$. This point is not quite stable in the sense that the adaptive landscape at $u^{*}=0$ is neither a minimum nor a maximum. The landscape has gone flat. But the moment $\sigma_{k}^{2}>\sigma_{a}^{2}$ the two species' strategies diverge and their equilibrium populations become less than $K_{m}$ but greater than $\frac{K_{m}}{2}$ (figure $3 \mathrm{~B}$ ). Hence the sum of the two species' total population sizes is now greater than the size $K_{m}$ of the single species equilibrium.

As the difference between $\sigma_{k}^{2}$ and $\sigma_{a}^{2}$ becomes larger and goes towards infinity the equilibrium values for the two species' strategies diverge towards infinity, and their population sizes increase towards $K_{m}$ meaning the total population size of the community increases towards $2 * K_{m}$. Furthermore, both the difference and ratios of $\sigma_{k}^{2}$ and $\sigma_{a}^{2}$ directly influence the 
species' equilibrium strategies and population sizes. Hence, there are an infinite number of combinations of $\sigma_{k}^{2}$ and $\sigma_{a}^{2}$ that will produce the same $u^{*}$ and this set would appear as a curved line in the state space of $\sigma_{k}^{2}$ and $\sigma_{a}^{2}$. Similarly, there are an infinite number of combinations of $\sigma_{k}^{2}$ and $\sigma_{a}^{2}$ that will produce the same $x^{*}$. However, combinations that produce the same equilibrium strategy values will not produce the same equilibrium population sizes, and vice versa for combinations that produce the same equilibrium population sizes.

The simulations exactly match these results for the model parameters, $\sigma_{a}^{2}$ and $\sigma_{k}^{2}$, chosen in figure 3. For arbitrary initial conditions, simulations of the two-species Darwinian dynamics always converged on the predicted two-species equilibria. The simulations suggest that there are no non-symmetric two-species equilibria and, indeed, the analytic proof of this appears in the Appendix B for arbitrary model parameters.

Finally, for this L-V competition model, it is noteworthy that the two species' strategies both converge on minima of the adaptive landscape (figure $3 \mathrm{C}$; see the Appendix $\mathrm{C}$ for a proof of this result). In particular, $\left(\mathbf{u}^{*}, \mathbf{x}^{*}\right)$ is not an ESS according to Definition 1. Thus, we can expect each of these species to undergo adaptive speciation to produce a new four species community. The next section will explore what happens at and after a four-species equilibrium.

\section{Infinite Niche Packing}

In this section we first describe a species doubling algorithm to demonstrate the convergence of evolution to a continuum of strategies. The associated analytic computations for the equilibria beyond two strategies are intractable and thus computer simulations were used. The default parameters for these simulations are $\sigma_{k}^{2}=200, \sigma_{a}^{2}=4$ and $K_{m}=100$. We use Darwinian dynamics for these simulations. The description of these computer based simulations and the corresponding results will be followed with a description of the end 
point of these speciation events.

\section{Infinite Niche Packing through Species Doubling}

There are three ways we can seek eco-evolutionary solutions for the L-V model when species diversity is greater than two $(n>2)$. The first would be to numerically solve for $\mathbf{u}^{*}$ and $\mathbf{x}^{*}$ from the $2 n$ simultaneous equations representing the first order necessary conditions for evolutionary and ecological stability, respectively. The advantage of this approach is that it can find all singular points on the adaptive landscape whether these strategies and population sizes are actually stable or not under the Darwinian dynamics. For instance, a given $u_{i}^{*}$ may reside on a peak of the landscape (satisfying $\frac{\partial G}{\partial v}=0$ ) and could be one component of an ESS. But, this peak may not be convergent stable in the sense that a species starting with a strategy near the peak will evolve away from $u_{i}^{*}$ rather than converging on that strategy. This uncertainty then also becomes the disadvantage of the approach. One must still run the Darwinian dynamics to determine whether $\mathbf{u}^{*}$ and $\mathbf{x}^{*}$ will be convergent stable and likely outcomes of natural selections.

This leads to a second way for finding eco-evolutionary solutions by simulating Darwinian dynamics on a system seeded with $n$ preselected strategy values and associated population sizes. The disadvantage of this approach is that it may never converge either due to nonequilibrial evolutionary and/or ecological dynamics, or because an actual solution is not dynamically accessible from the initial conditions. For instance, even if there is an $n$ species solution, the initial condition may result in various pairs of species converging on the same $u^{*}$ effectively reducing the number of distinct species. The outcome can be highly sensitive to initial conditions.

A third way to seek eco-evolutionary solutions is to let the number of species be dynamic as well. This can be done by starting the system with one (or more) species. If Darwinian 
dynamics evolve to a convergent stable minimum, then induce adaptive speciation by introducing two progeny species each with distinct strategies that are very close in value to the progenitor species (this can usually be done by adding and subtracting a small $\delta$ from the original strategy), and letting the new species start with a small population size. Darwinian dynamics then resume with these two species. If one or both of the progeny species converge on minima then continue the process of adaptive speciation for those species. This approach permits a gradual buildup of species from a single common ancestor. So long as the Darwinian dynamics converge on niche archetypes, then the process will continue indefinitely unless a level of species diversity has been reached where all of the species' strategies reside on peaks of the adaptive landscape. A disadvantage of this approach is that it may stop at a species diversity that is lower than could occur at other eco-evolutionary solutions (e.g., Vincent and Brown 2005, Ripa et al. 2009).

For simulating the L-V model, we chose the third approach while variously exploring the model with all three approaches. The details on how the simulations were conducted are given in the Appendix D. Quite often convergence was very slow in the neighborhood of the equilibrium points. Using these simulations, we saw that there was a doubling of species each time a niche archetype equilibrium was reached as far as 64 species (i.e. from 1 to 2 , to 4 , to 8 , to 16 , to 32 , to 64 ). These simulations produced dynamics illustrate several results. First, the strategy dynamics produce the actual phylogeny of strategy evolution over time. Here, we see that distance between the strategies of each species gets smaller and smaller as more species are added (figure $4 \mathrm{~A}$ ). Second, it shows how the equilibrium population size of each species decreases with each increase in diversity (figure $4 \mathrm{~B}$ ). The amount of time required to reach equilibrium also increases with diversity. With $k=1$, the two species equilibrium is reached in approximately two thousand time steps, while it takes more than thirty thousand to go from eight to sixteen species.

The complete eco-evolutionary dynamics resulting in 1024 species in about 3.5 million 
time steps are obviously impossible to plot legibly. However, at each convergent stable minimum, we can summarize the range of the equilibrium strategies (figure $5 \mathrm{~A}$ ), the range of the population sizes (figure $5 \mathrm{~B}$ ) and the productivity of the community (total population size of all species combined, figure $5 \mathrm{C}$ ).

We make several conjectures based on these simulations. First, as the diversity increases to infinity, the maximum and minimum strategies also go to $+/$ - infinity, respectively, but the distance between adjacent species decreases to the point of being infinitely small at infinite diversity (figure 5 A). Second, when diversity is infinite, population sizes are infinitely small (figure 5 B). Finally, even when diversity is infinite, there is a finite limit to the productivity of the community imposed by the niche width. To strengthen these conjectures, we ran the simulation again up to 1024 species using a different set of parameters which took about 3.2 million time steps (figure $5 \mathrm{D}-\mathrm{F}$ ). The range of equilibrium strategies increased more slowly with community diversity, and the maximum productivity of the community was lower, but our three conjectures remain.

\section{Infinite Niche Packing through Nash equilibrium}

The adaptive speciation events from the previous section (up to $2^{10}=1024$ species), are anticipated to continue indefinitely. Thus, we expect this basic L-V competition model to support infinitely many species. Moreover, since the distance between adjacent species for the $n$-species symmetric equilibrium declines towards 0 and the overall distance between the most extreme niche types appears to become arbitrarily large as $n$ increases (see figure $4,5)$, the limiting process will produce infinite niche packing in each subinterval of the strategy axis. In fact, we expect the limiting population sizes (or relative frequencies) will be a continuous distribution $P^{*}(u)$ along the strategy axis. That is, $P^{*}(u)$ is positive for all $u$ and so $P^{*}$ is a distribution with full support, meaning the continuum of $u$ coexists in the 
population. From game theory, the stability of $P^{*}$ would imply that this distribution must be a Nash equilibrium (NE). Otherwise, some species will have a higher fitness and so be able to invade, thus destabilizing the system.

Generalizing the G-function approach to distributions, we take

$$
G(v, \mathbf{u}, P)=\frac{r}{K(v)}\left[K(v)-\int_{-\infty}^{\infty} a(v, u) P(u) d u\right]
$$

as the fitness of the focal strategy $v$. When $P$ is a distribution with a finite number of species, $\mathbf{u}=\left(u_{1}, \ldots, u_{n}\right)$, this integral reduces to the original $n$-species G-function (1). For notational convenience more in line with that used in game theory, since $P$ already specifies the niche types $u$, we denote $G(v, \mathbf{u}, P)$ simply as $\pi(v, P)$ and call it the expected payoff to an individual using strategy $v$ when the population distribution is $P$.

Since $P^{*}$ is at an ecological equilibrium with full support, it is a solution to

$$
\pi(v, P)=0 \text { for all niche types } v \text {. }
$$

One such distribution, which has been known since this model was introduced by Roughgarden (1979) (see also Barabás et al. 2012), is given by the normal distribution

$$
P^{*}(u)=\frac{K_{m} \sigma_{k}}{\sigma_{a} \sqrt{2 \pi\left(\sigma_{k}^{2}-\sigma_{a}^{2}\right)}} e^{-u^{2} /\left(2\left(\sigma_{k}^{2}-\sigma_{a}^{2}\right)\right)}
$$

adjusted by the equilibrium total population size $\int_{-\infty}^{\infty} P^{*}(u) d u=K_{m} \frac{\sigma_{k}}{\sigma_{a}}$. For the parameters used in figure 5 , the population sizes are $100 \sqrt{\frac{200}{4}}=100 \sqrt{50}=707.107$ (figure $5 \mathrm{C}$ ), and $100 \sqrt{\frac{50}{4}}=50 \sqrt{50}=353.553$ (figure $5 \mathrm{~F}$ ) which are in almost perfect agreement with the simulations with up to 1024 species. It can be shown that for the $P^{*}$ given in Equation (15), $\pi\left(v, P^{*}\right)$ is independent of $v$ and so a Nash equilibrium (see Appendix E).

It is likely that this distribution $P^{*}$ is global and unique for a given set of parameters. 
If this was not so, then any convex combination of $P^{*}$ and an arbitrary $P$ would also be a $\mathrm{NE}$, and so $P^{*}$ would not exhibit its observed convergence and stability in our simulations. If these solutions were not unique, we would expect some of our simulations to converge on different equilibria depending on initial conditions.

The NE concept is also relevant for the $n$-species equilibrium. It is then defined as a strategy vector $\mathbf{u}^{*}=\left(u_{1}^{*}, \ldots, u_{n}^{*}\right)$ such that no individual has a unilateral incentive to change its strategy, with the added feature that the payoff to each species at the $n$-species equilibrium is 0 (ecological equilibrium). Since there is a single payoff function for all species (i.e. a single Gfunction), the NE requirement is:

$$
G\left(v, \mathbf{u}^{*}, \mathbf{x}^{*}\right) \leq G\left(u_{i}^{*}, \mathbf{u}^{*}, \mathbf{x}^{*}\right) \text { for all } v \quad i=1, \cdots, n
$$

For the basic L-V game with competition between $v$ and $u_{i}$ given by $a\left(v, u_{i}\right)$, there is no $n$-species NE since $\left(\mathbf{u}^{*}, \mathbf{x}^{*}\right)$ is a convergent stable minimum; $G\left(v, \mathbf{u}^{*}, \mathbf{x}^{*}\right)>G\left(u_{i}^{*}, \mathbf{u}^{*}, \mathbf{x}^{*}\right)$ for all $v$ not equal to one of the niche archetypes $u_{i}^{*}$ (figures $3 \mathrm{C}$ and $4 \mathrm{C}$ )). This inequality is the root cause of the speciation events for each species $i$ in the section "Infinite Niche Packing through Species Doubling".

\section{Modifications of competition and carrying capacity}

Barabás et al. (2012) and references therein noted how altering the functional forms of the carrying capacity and competition functions likely result in ESSs with a finite number of species. In these sections we examine two modifications that collapses infinite niche packing. We can arrive at analytic solution for two species and computer simulations for greater numbers of species. We first consider a modification to the competition function followed by a modification to carrying capacity. 


\section{The effect of baseline competition}

Suppose there is some baseline competition between all individuals no matter how distant their strategy values. This makes ecological sense because closely related species (those from the same Gfunction) may have certain shared resource requirements no matter how distinct their strategies. In addition to the unavoidable baseline competition we add back our function that has like competing most with like. We model this by replacing $a\left(v, u_{i}\right)=$ $\exp \left[-\frac{\left(v-u_{i}\right)^{2}}{2 \sigma_{a}^{2}}\right]$ with

$$
p+(1-p) a\left(v, u_{i}\right)
$$

in the G-function (1) (figure $1 \mathrm{C}$ ). Here $0 \leq p<1$ represents the relative weight of these two competition factors with $p=0$ giving the original model and, as $p$ increases, the effect of the baseline competition becomes more pronounced (we exclude the degenerate case $p=1$ which corresponds to equal competition between all individuals). The single species equilibrium remains at $u^{*}=0$ with equilibrium population size $x^{*}=K_{m}$. More interestingly, if baseline competition is sufficiently high (in fact $p>p_{1}^{*} \equiv 1-\frac{\sigma_{a}^{2}}{\sigma_{k}^{2}}$ as shown in the Appendix F), then this equilibrium is at a maximum of its adaptive landscape (see figure 6 where $p_{1}^{*}=0.98$ for our default parameters). In fact, it is now a single species ESS as well as an NIS, and thus automatically convergent stable (Apaloo 1997). Furthermore, it appears to be the global outcome of Darwinian dynamics no matter how many species are initially present (figure 6).

For lower amounts of baseline competition (i.e. $0 \leq p<p_{1}^{*}$ ), adaptive speciation occurs at $u^{*}=0$. The resultant two-species symmetric equilibrium is now (see Appendix F) 


$$
\begin{aligned}
u^{2} & =\frac{\sigma_{a}^{2}}{2} \ln \left(\frac{2 \sigma_{k}^{2}-\sigma_{a}^{2}}{\sigma_{a}^{2}} \frac{1-p}{1+p}\right) \\
x & =K_{m} \frac{1}{1+p} \frac{\sigma_{a}^{2}}{2 \sigma_{k}^{2}}\left(\frac{2 \sigma_{k}^{2}-\sigma_{a}^{2}}{\sigma_{a}^{2}}\right)^{1-\frac{\sigma_{a}^{2}}{4 \sigma_{k}^{2}}} .
\end{aligned}
$$

It is of interest to identify analytically the nature of this two-species equilibrium as $p$ varies on the interval $\left[0, p_{1}^{*}\right]$. Here, we only consider results obtained from computer simulations. The equilibrium is a convergent stable ESS according to Definitions 1 and 2 with $n=2$ for $p_{2}^{*}<p<p_{1}^{*}$ where $p_{2}^{*}=0.93$ for the default parameters ( $p_{2}^{*}$ was found by simulation). For $0 \leq p<p_{2}^{*}$, the two-species equilibrium is a convergence stable minimum and so speciation can occur at both. However, the initial four species reduce to three for $p$ just a bit less than $p_{2}^{*}$ (figure $6 \mathrm{~A}$ ) since the two new species closest to $u=0$ eventually evolve to $u=0$ and form one niche type in an ESS coalition of three.

These numerical simulations for our default parameters were used in two separate ways. First, with the number of species fixed at two, the simulations return the same convergence stable minima as in figure 3 when $p=0$ (see also figure $6 \mathrm{~A}$ ) where $u_{1}^{*}=3.03$ and $u_{2}^{*}=-3.03$. As $p$ increases, strategy values tend to 0 from both sides (figure $6 \mathrm{~A}$ ) and the equilibrium population size declines (figure 6 B). As $p$ approaches 1, the two-species equilibrium becomes a convergent stable ESS when $p$ reaches 0.93 and then disappears for $p>0.98$ when a singlespecies ESS at $u^{*}=0$ emerges.

The second use of these numerical simulations examines how increases in the level of baseline competition alters the speciation events discussed in section "Infinite Niche Packing through Species Doubling" up to 16 species in figure 4 and 1024 species in figure 5 . That is, we ran the simulations as in section "Infinite Niche Packing through Species Doubling", but with different baseline levels of competition, $p$, to see how at the ESS the total number of 
species, the maximum range of strategies, and the total community productivity changed. As predicted, in the absence of baseline competition (i.e. $p=0$ ), the simulations return the same result as the original simulations for 1024 species (shown in figure $5 \mathrm{~A}-\mathrm{C}$ ). However as $p$ increases, the total number of unique equilibrium strategies (i.e. species) rapidly declines and corresponds to a convergent stable ESS. Indeed, with $p=0.01$, the system already collapses to just 52 unique species (figure $7 \mathrm{~A}$ ), compared to infinitely many strategies at $p=0$. As $p$ increases further, the maximum and minimum strategy values converge on zero, and the community productivity converges on $K_{m}$ (figure $7 \mathrm{~B}, \mathrm{C}$ ). Eventually, the system collapses to a two-species convergent stable ESS $(0.93<p<0.98)$ and then to a single-species ESS $(0.98<p<1)$ (figure $7 \mathrm{~A})$.

The simulations constraining the system to two species (figure $6 \mathrm{~A}, \mathrm{~B}$ ), or to at most 1024 species (figure $7 \mathrm{~A}-\mathrm{C}$ ) produce consistent conclusions: any ecological factors that do not allow species to evolve to strategies that produce zero competition among some members of the community, but instead introduce some minimum level of baseline competition will cause the system to go from infinite species to some finite number.

By imposing a baseline level of competition between all individuals, the equilibrium population sizes of any convergent stable point with more than two species will be less than that of the original model. This is because for any given pair of species (with different strategy values) their competition coefficient will be larger than for original model..

\section{The effect of truncated trait space}

In the L-V model of section "An Eco-Evolutionarry Lotka-Volterra Model" the carrying capacity function insures that all values of $v$ will result in $K(v)>0$. Szabo and Meszena (2006) showed how changing the carrying capacity function from Gaussian can collapse unlimited niche packing down to just a few species. Gyllenberg and Meszena (2005) and 
Barabas et al. (2012) achieved this through a truncated niche space. In this section, we provide an eco-evolutionary exploration of the consequences of limiting the range of strategy values that support a positive carrying capacity. To do this, we replace $K(v)$ in (3) by a carrying capacity that still has maximum $K_{m}$ at $u=0$ but no carrying capacity outside an interval centered at $u=0$ (figure $1 \mathrm{D}$ ). Specifically, we take carrying capacity as

$$
K(v)+s\left(K(v)-K_{m}\right)
$$

whenever this expression is positive and 0 otherwise. Here $s$ is a positive constant that influences the range of strategies that result in a positive carrying capacity. As shown in Appendix G, carrying capacity is positive for $v^{2}<2 \sigma_{k}^{2} \ln \left(\frac{1+s}{s}\right)$, an interval that becomes smaller as the "shrinking" parameter $s$ increases (figure $1 \mathrm{D}$ ). The following results are also proved in the Appendix G.

When $s$ is close to 0 , the single species equilibrium at $u^{*}=0$ undergoes adaptive speciation as in the original model except that the resulting two-species symmetric equilibrium now has trait values $\pm u_{1,2}^{*}$ where $u_{1,2}^{* 2}$ is the unique solution for $u^{2}$ in

$$
e^{2 u^{2} / \sigma_{a}^{2}}=\frac{2 \sigma_{k}^{2}-\sigma_{a}^{2}}{\sigma_{a}^{2}}-\frac{s}{1+s} \frac{2 \sigma_{k}^{2}}{\sigma_{a}^{2}} e^{u^{2} / 2 \sigma_{k}^{2}}
$$

(cf. (13)). There is a two-species symmetric niche archetype if and only if the shrinking parameter $s$ is below the threshold value

$$
s^{*}=\frac{\left(\sigma_{k}^{2}-\sigma_{a}^{2}\right)}{\sigma_{a}^{2}} .
$$

For $s$ above this threshold value there is no two-species symmetric equilibrium and the single species equilibrium $u^{*}=0$ is an ESS and NIS (and thus convergent stable) that appears to be the eventual outcome of the combined eco-evolutionary dynamics (4) and (5) from any 
arbitrary initial condition.

As in the preceding section, we consider only the results from simulations regarding the nature of evolutionary stability of the two-species symmetric equilibrium as $s$ varies on the interval $\left[0, s^{*}\right)$. The simulations did not reveal a second threshold value of $s$, say $s_{2}^{*}$ such that the two-species equilibrium is an ESS (i.e. has no further speciation) for any $s$ in the interval $\left(s_{2}^{*}, s_{1}^{*}\right)$ with $s_{1}^{*}=s^{*}$,

We used our numerical simulations to explore the effect of truncating trait space in the same ways that we did for baseline competition. The results are broadly similar. First, for two species when $s=0$, the simulations return the same result as the original model (shown in figure 3 ) such that $u_{1}=3.03$ and $u_{2}=-3.03$ (figure $6 \mathrm{C}$ ). As $s$ increases and the trait space becomes smaller and smaller, the equilibrium strategy value declines, as does the equilibrium population size, eventually resulting in a single species with a strategy that is a convergent stable ESS instead of a convergent stable minimum on the adaptive landscape (figure $6 \mathrm{C}, \mathrm{D}$ ). Second, we examined how truncating the trait space would alter the solutions for 1024 species. For this analysis, we ran the simulations as in section "The Lotka-Volterra Competition Game with Two Species", and as for the section "The effect of baseline competition" but with varying values of trait space truncation, $s$, to see how the total diversity changed, how the maximum range of strategies changed, and how total community productivity changed. When $s=0$, the simulations return the same result as the original simulations of 1024 species (shown in figure $5 \mathrm{~A}-\mathrm{C}$ ). As $s$ increases, the total number of unique equilibrium strategies (i.e. species) rapidly declines, eventually collapsing to just one species (figure $7 \mathrm{~A}$ ). Similarly, as $s$ increases, the maximum and minimum strategy values converge to zero, and the community productivity converges to $K_{m}$. The simulations for truncating trait space where the system is constrained to two species (figure $6 \mathrm{C}, \mathrm{D}$ ), or to 1024 species (figure $7 \mathrm{D}-\mathrm{F}$ ) produce identical conclusions: any ecological factors that truncate the trait space will cause the system to go from infinite species to some finite number 
the achieve and ESS. Indeed, with $s=0.01$ the system collapses to an ESS of just 8 unique species, compared to infinitely many strategies at $s=0$ (figure $7 \mathrm{D}$ ).

By truncating the carrying capacity function, the equilibrium population sizes of any convergent stable point with more than two species will be less than that of the original model. This is because once a species strategy deviates from $u=0$, its carrying capacity declines more rapidly in the this truncated trait space model than in the original.

\subsection{A Quartic Competition Function}

Leimar et al (2013) investigated the properties of communities based upon the L-V unlimited niche packing model, but with a quartic version of the competition function:

$$
a\left(v, u_{i}\right)=\exp \left[-\frac{\left(v-u_{i}\right)^{4}}{2 \sigma_{a}^{2}}\right]
$$

They found a more or less regular spacing of species with possibility of adding species at either end of the niche axis. While providing for unlimited niche packing, the coexisting species tended to reside near peaks rather than valleys of the adaptive landscape. We can run their model using the ecological and evolutionary dynamics. A single species with any starting strategy value will evolve to a peak at $u^{*}=0$. However, this peak is only a locally convergent stable maxima. Across valleys in the adaptive landscape their exist strategies to the left and to the right that would be able to invade. The valleys preclude adaptive speciation as happens at the convergent stable minima. To create a faunal buildup new species must be added some distance from the resident species. Upon adding species across the valleys of the adaptive landscape, the new, more diverse community evolves until all species reside on local maxima, with valleys at each end of the niche axis providing evolutionary barriers to additional species with still higher fitness (see Fig. X for an example with a convergent stable community of 11 species). In accord with Leimar et 
al. (2013), the species' strategies are more or less uniformly spaced. This model provides a nice starting point for investigating faunal buildup when unlimited niche packing cannot be achieved through speciation at convergent stable minima. As before, one could adjust the competition function to reveal ESSs with finite numbers of species.

\section{Discussion}

We opened with Hutchinson's (1959) question: "why are there so many species?" We suggest that this is because previous ideas about competitive exclusion and limiting similarity generally predict fewer species than we observe in natural systems (Chesson 2000; Silvertown 2004). Indeed, Hutchinson (1961) later went so far as to call this discrepancy between theory and observation a "paradox". In general, previously published evolutionary games also predicted that natural selection would produce a finite number of strategies. These outcomes occur under both discrete or continuous niche axes. Starting with Roughgarden (1979) and continuing with others (Bulmer 1974; Sasaki and Ellner 1995; Sasaki 1997; Gyllenberg and Meszéna 2005; Meszéna et al. 2006; Szabó and Meszéna 2006; Barabás and Meszéna 2009; Parvinen and Meszéna 2009; Barabás et al. 2012; Barabás et al. 2013; D’Andrea et al. 2013) it is been shown how unlimited niche packing is in theory feasible, but robust to slight alterations of the carrying capacity and competition functional forms. Our eco-evolutionary extension of these works shows how a finite number of species will evolve to convergent stable equilibria that we describe as "niche archetypes". These various eco-evolutionary equilibria are not haphazard collections of species but structured with distinct and well organized patterns of abundances and strategy values. Furthermore, these convergent stable configurations result in very specific patterns of trait spacing among the coexisting species (not unlike

Hutchinson's ratios). We examine how faunal buildup from a few to many species (we went as far as 1024) can occur as adaptive speciation (evolutionary branching) from convergent 
stable minima of the adaptive landscape. Interestingly, when the competition function is raised to the 4th power rather than simply squared (Leimar et al. 2013), unlimited niche packing still occurs, but all of the niche archetypes for a finite number of species represent non-ESS, convergent stable maxima (local maxima, only). It has been noted that models with unlimited niche packing collapse to finite numbers of species with slight changes to the form of the competition function. We see the same, and with eco-evolutionary dynamics faunal buildup or the invasion of species with distinct strategies can lead to the ESS.

In the L-V model we analyzed, if the width of the niche axis $\left(\sigma_{K}\right)$ is less than the competition coefficient $\left(\sigma_{a}\right)$, it is known that the outcome of evolution is a single strategy that is a convergent stable ESS (figure $2 \mathrm{~A}-\mathrm{C}$ ). When the inequality is reversed, the result no longer holds. Our numerical and analytical results are compatible, and show that species doubling can continue indefinitely, with each doubling of the number of species occurring after the Darwinian dynamics have reached convergent stable minima based on a coalition of $2^{n}$ strategies (figures 4 and 5). Due to constraints of computing power, even with a supercomputer cluster, the maximum number of strategies attained in the simulations was 1024. To run a single simulation past this number of strategies would take excessive computational time (>weeks), and require enormous amounts of storage space for the output (>900GB). However, several results were evident by the time simulations arrived 1024 species. These include: (1) the spacing between the niche archetypes decline as the number of species increase; (2) the range of the species in the convergent stable strategy coalitions increased as the number of species increased; (3) the total population size approached a limiting value (figure 5). The third result is particularly interesting. It is almost in perfect agreement with the value obtained in Eqn 15 by an analytic limit argument. As the number of species increases, the simulations showed that the distribution of convergent stable strategies converges on the Nash distribution (Eqn 15). In sum, that our analytic proofs based on infinite diversity asymptote with our simulations based on 1024 species leads us to conclude that in- 
finite diversity by species doubling is theoretically possible. As diversity approaches infinity, there is also no longer any limiting similarity: species differences also approach zero (figure $4 \mathrm{~A}$ ). We concur with many of the others cited above that the L-V model of unlimited niche packing is structurally non-robust, but it provides a valuable ideal from which to develop and contrast models predicting a finite number of species at the ESS.

Gyllenberg and Meszéna (2005) and Meszéna et al. (2006) demonstrated the nonrobustness of the coexistence of a continuum of species or phenotypes in a large class of models including the L-V model used here. Gyori Barabás and colleagues in a series of elegant papers (Barabás and Meszéna 2009; Barabás et al. 2012; Barabás et al. 2013) examine the structural robustness of several forms of the competition equation. In particular, Barabás et al. (2012) start with a number of forms that generate infinite niche packing and then show how 400 or so species will collapse to communities of 5 -10 upon small perturbations to the shape of the competition function (their Figs 4, $5 \& 7$ ). The ecological dynamics and results of our work represent examples of these results. In addition to the ecological dynamics of fixed species, we consider the model as an evolutionary game, and consider the evolutionary dynamics that traverse frequency-dependent and highly dynamic adaptive landscapes. When the game permits a continuum of species, any finite number of species evolved to distinct, convergent stable minima from which adaptive speciation can continue the diversity buildup. Similarly, when the competition curve or carrying capacity functions were modified, a convergent stable ESS with a fixed number of species residing on peaks of the adaptive landscape emerged. In all of our examples, the eco-evolutionary dynamics of the game led to very specific niche positions of the coexisting species whether the number of species was at or below the number of species in the ESS coalition.

We considered two modifications of the L-V model to study what evolutionary factors might produce a finite rather than an infinite number of species. Here, analytic results are available for only one species or two species and simulation analyses were used to provide 
further insights into the outcomes of the eco-evolutionary dynamics. These numerical results are again in agreement with the analytic results when there are a small number of species.

First, we considered a modification of the competitive interaction to include a minimum level of baseline competition by introducing a parameter that varied between 0 and 1 (figure $1 \mathrm{C})$. Concerning the two species solution, we observe that as the baseline competition parameter increases from 0 to 1 , there is an initial regime where the two strategies form a convergent stable minimum, followed by a regime of a convergent stable ESS before a final collapse of the two species solution to a single species convergent stable ESS solution(figure 6 A, B). These simulation results agree with the results predicted by the analytic calculations. In the case of 1024 species solution, we observe that, as the parameter increases from 0 , the number of species in the equilibrium solutions declines rapidly to a solution with just one species (figure $7 \mathrm{~A}$ ). We propose that, any ecological factors that do not allow species to evolve to strategies that produce exactly zero competition among some members of the community, but instead introduce some level of baseline competition will cause the system to go from infinite species to some finite number depending on the intensity of the baseline competition. That is, with a minimum baseline level of competition, there will be a limit to how similar species can be and still coexist. For example, all plants require the same set of limiting nutrients in approximately the same proportions (Knecht and Goransson 2004; Tilman 1982). In a forest, the level of competition that occurs between a canopy tree and a small annual herb will be so small as to be almost certainly impossible to measure. Yet, every nutrient ion that is captured by the tree is one less ion that is available to the herb and vice versa. This means that there is a tiny but finite level of baseline competition that necessarily occurs between all cooccurring plants. It is noteworthy that such small levels of competition will be ecologically unimportant within the ESS community. However, these small levels of competition are paramount in maintaining a finite number of species at the ESS, and in imposing a limit to how similar species can be and still coexist (figure 7 A). No 
matter how small, a finite baseline level of competition in the L-V model collapses unlimited niche packing into a finite number of species.

Second, we truncated carrying capacity so that only trait values in a finite symmetric interval about 0 can support populations. The truncation is measured as a shrinkage parameter which, ecologically, constrains the trait space to some finite interval (figure $1 \mathrm{D}$ ). The results obtained here are similar to those for the case with baseline competition. As the parameter increased from 0 it increasingly constrained the trait space, and above some threshold value, the two species solution collapsed to a one species equilibrium that is a convergent stable ESS (figure 6 C, D). When we start the simulation with 1024 species, as the shrinkage parameter increases (i.e. the niche width decreases), the number of species in a convergent stable ESS declines and eventually a solution of one species is obtained (figure 7 D). From these simulations, we propose that any ecological factors that truncate the trait space will cause the system to go from infinite species to some finite number with increasing shrinkage parameter values. Returning to our forest example, plant height is a critically important trait within evolutionary games played by plants (Dybzinski et al. 2011; Falster and Westoby 2003; Givnish 1982). Taller plants are able to gain access to sunlight, and leave shorter plants struggling to fix carbon in the shade; plant competition above-ground is clearly an arms race for height. However, plants are only able to move water up their stem by passive evapotranspiration. This means that the Earth's gravity imposes a strict limit on how high passive evapotranspiration can lift water, and constrains the trait space to a maximum height of 122-130 metres in the absence of mechanical damage (Koch et al. 2004). Again, our analysis suggests that such constraints are enough to limit the system to some finite number of species at a convergent stable ESS.

While unlimited niche packing is theoretically possible, we are not suggesting that there are an infinite number of species on Earth. This is demonstrably false. But, if infinitely many species are theoretically possible, but not actually achieved, it begins to turn the ques- 
tion of "why are there so many species?" on its head. Based on the analyses presented in this paper we ask: "why are there so few species?". We have provided situations with more realistic competition interaction (baseline competition) and carrying capacity (truncated trait space) that reduce unlimited niche packing to a finite number of evolutionarily coexisting species. Previous theories of biodiversity generally predict fewer species than are observed in real systems, while the (unmodified) L-V evolutionary game predicts more species than are observed in real systems. The truth, and the solution to Hutchinson's (1961) "paradox", is obviously somewhere in the middle. We suggest two immediate empirical questions that could begin to find this middle based on baseline competition and the size of the trait space. It is noteworthy that with these modifications diversity drops from literally 1000's to 5's and 10's. The drastic drop in diversity seen by introducing a fixed baseline level of competition may also under-represent species diversity. Likely factors increasing diversity (and compatible with these models) include multiple niche axes (rather than just one), and geographically separated communities that evolve somewhat different species that may be more or less ecological equivalents in space.

Different taxonomic groups likely exhibit different ecologically permissible ranges of their trait space due to different biophysical constraints different evolutionary trade-offs for trait evolution. Do taxonomic groups with fewer trait constraints correspond with those taxonomic groups with high observed levels of coexisting species? For example, the insect Order Coleoptera is extremely diverse compared to other insect Orders, and indeed other taxa more broadly. Is there something about Coleopterans that introduces either less competition among functionally similar taxa, or introduces fewer constraints on their morphological or physiological trait space? Similarly, the mammal Family Felidae contains relatively few species globally, and rarely more than a few species of Felidae coexist locally. Is there something about felines that introduces more competition among functionally similar taxa, or introduces more constraints on morphological and physiological trait space? These are two 
extreme examples, but the constraints on competition and traits among organisms from microbes to whales are probably very different and may provide clues as to why there are so many and yet so few species on earth. In summary, the diversity of life on earth may be a finite and greatly reduced subset of what is evolutionarily feasible and ecologically acceptable.

\section{Acknowledgement}

We thank Rosalyn Rael for valuable discussion and comments. Four reviewers provided helpful comments and insights for revising earlier drafts. The research was supported by The Dr. W.F. James Chair of Studies in the Pure and Applied Sciences at St. Francis Xavier University. JSB and RC both appreciate the opportunity of funding from their Chair positions which was instrumental in the successful completion of this project. This research was partially funded by the Natural Sciences and Engineering Research Council (NSERC) of Canada through a Banting Post-Doctoral Fellowship (GGM) and an Individual Discovery Grant (RC). This material is based upon work supported by the National Science Foundation Graduate Research Fellowship (AH) under Grant Nos. DGE-0907994 and DGE-1444315. Any opinion, findings, and conclusions or recommendations expressed in this material are those of the authors and do not necessarily reflect the views of the National Science Foundation.

\section{References}

Abrams, P. A., Matsuda, H. \& Harada, Y. (1993), 'Evolutionarily unstable fitness maxima and stable fitness minima of continuous traits', Evolutionary Ecology 7(5), 465-487. 
Apaloo, J. (1997), 'Revisiting strategic models of evolution: The concept of neighbourhood invader strategies', Theoretical Population Biology 52, 71-77.

Apaloo, J. (2003), 'Single species evolutionary dynamics', Evolutionary Ecology 17(1), 33-49.

Apaloo, J., Brown, J. S. \& Vincent, T. L. (2009), 'Evolutionary game theory: ESS, convergence stability, and NIS', Evolutionary Ecology Research 11, 489-515.

Barabás, G., DâĂŹAndrea, R. \& Ostling, A. M. (2013), 'Species packing in nonsmooth competition models', Theoretical ecology 6(1), 1-19.

Barabás, G. \& Meszéna, G. (2009), 'When the exception becomes the rule: the disappearance of limiting similarity in the lotka-volterra model', Journal of theoretical biology 258(1), 89-94.

Barabás, G., Pigolotti, S., Gyllenberg, M., Dieckmann, U. \& Meszéna, G. (2012), 'Continuous coexistence or discrete species? a new review of an old question', Evolutionary Ecology Research 14(5), 523-554.

Brown, J. S. \& Pavlovic, N. B. (1992), 'Evolution in heterogeneous environments: Effects of migration on habitat specialization', Evolutionary Ecology 6, 360-382.

Brown, J. S. \& Vincent, T. L. (1987), 'A theory for the evolutionary game', Theoretical Population Biology 31, 140-166.

Brown, J. S. \& Vincent, T. L. (1992), 'Organization of predator-prey communities as an evolutionary game', Evolution 46, 1269-83.

Bulmer, M. (1974), 'Density-dependent selection and character displacement', American Naturalist pp. $45-58$. 
Chesson, P. (2000), 'Mechanisms of maintenance of species diversity', Annual review of Ecology and Systematics 31, 343-366.

Christiansen, F. B. (1991), 'On conditions for evolutionary stability for a continuously varying character', American Naturalist 138, 37-50.

Cohen, Y., Vincent, T. L. \& Brown, J. S. (1999), 'A g-function approach to fitness minima, fitness maxima, evolutionary stable strategies and adaptive landscapes', Evolutionary Ecology Research 1, 923-942.

Cressman, R. (2010), 'Css, nis and dynamic stability for two-species behavioral models with continuous trait spaces', Journal of theoretical biology 262(1), 80-89.

D’Andrea, R., Barabás, G. \& Ostling, A. (2013), 'Revising the tolerance-fecundity trade-off; or, on the consequences of discontinuous resource use for limiting similarity, species diversity, and trait dispersion*', The American Naturalist 181(4), E91-E101.

Doebeli, M. \& Dieckmann, U. (2003), 'Speciation along environmental gradients', Nature 421(6920), 259-264.

Dybzinski, R., Farrior, C., Wolf, A., Reich, P. B. \& Pacala, S. W. (2011), 'Evolutionarily stable strategy carbon allocation to foliage, wood, and fine roots in trees competing for light and nitrogen: an analytically tractable, individual-based model and quantitative comparisons to data', The American Naturalist 177(2), 153-166.

Eshel, I. \& Motro, U. (1981), 'Kin selection and strong evolutionary stability of mutual help', Theoretical Population Biology 19, 420-433.

Falster, D. S. \& Westoby, M. (2003), 'Plant height and evolutionary games', Trends in Ecology $\&$ Evolution 18(7), 337-343. 
Fisher, R. A. (1958), The genetical theory of natural selection, Dover Publications, Inc., New York.

Gause, G. F. (1931), 'The influence of ecological factors on the size of population', American Naturalist 2(696), 70-76.

Gause, G. F. (2003), The struggle for existence, Courier Corporation.

Geritz, S. A. H., Kisdi, E., Meszena, G. \& Metz, J. A. J. (1998), 'Evolutionarily singular strategies and the adaptive growth and branching of the evolutionary tree', Evolutionary Ecology 12, 35-57.

Givnish, T. J. (1982), 'On the adaptive significance of leaf height in forest herbs', American naturalist 120, 353-381.

Gyllenberg, M. \& Meszéna, G. (2005), 'On the impossibility of coexistence of infinitely many strategies', Journal of mathematical biology 50(2), 133-160.

Hutchinson, G. E. (1959), 'Homage to santa rosalia or why are there so many kinds of animals?', American naturalist pp. 145-159.

Hutchinson, G. E. (1961), 'The paradox of the plankton', American Naturalist 95, 137-145.

Knecht, M. R. \& Goransson, A. (2004), 'Terrestrial plants require nutrients in similar proportions', Tree Physiology 24, 447-460.

Koch, G. W., Sillett, S. C., Jennings, G. M. \& Davis, S. D. (2004), 'The limits to tree height', Nature 428(6985), 851-854.

Leimar, O. (2009), 'Multidimensional convergence stability', Evolutionary Ecology Research $\mathbf{1 1}(2), 191-208$. 
Lotka, A. J. (1927), 'Fluctuations in the abundance of species considered mathematically (with comment by v. volterra)', Nature 119, 12-13.

MacArthur, R. H. (1958), 'Population ecology of some warblers of northeastern coniferous forests', Ecology 39(4), 599-619.

MacArthur, R. \& Levins, R. (1967), 'The limiting similarity, convergence, and divergence of coexisting species', American naturalist pp. 377-385.

Meszéna, G., Gyllenberg, M., Pásztor, L. \& Metz, J. A. (2006), 'Competitive exclusion and limiting similarity: a unified theory', Theoretical Population Biology 69(1), 68-87.

Metz, J. A., Geritz, S. A. H., Meszena, G., Jacobs, F. J. A. \& van Heerwaarden, J. S. (1996), Adaptive dynamics, a geometrical study of the consequences of near faithful reproduction, in S. J. van Streint \& S. M. Verduyn-Lunel, eds, 'Stochastic and Spatial Structure of Dynamical Systems', Royal Academy of Arts and Sciences, North Holland, Amsterdam, pp. 120-160.

Morris, D. W. \& Lundberg, P. (2011), Pillars of evolution: fundamental principles of the eco-evolutionary process, Oxford University Press.

Parvinen, K. \& Meszéna, G. (2009), 'Disturbance-generated niche-segregation in a structured metapopulation model', Evolutionary Ecology Research 11(4), 651-666.

R Core Team (2013), 'R: A language and environment for statistical computing'. ISBN 3-900051-07-0.

URL: http://www.R-project.org/

Ripa, J., Storling, L., Lundberg, P. \& Brown, J. S. (2009), 'Niche co-evolution in consumerÜresource communities', Evolutionary Ecology Research 11, 305-323. 
Roughgarden, J. (1979), Theory of Population Genetics and Evolutionary Ecology: An Introduction, Macmillan, New York.

Sasaki, A. (1997), 'Clumped distribution by neighbourhood competition', Journal of Theoretical Biology 186(4), 415-430.

Sasaki, A. \& Ellner, E. (1995), 'The evolutionary stable strategy in fluctuating environments', Evolution 49, 337-350.

Silvertown, J. (2004), 'Plant coexistence and the niche', Trends in Ecology ES Evolution 19(11), 605-611.

Szabó, P. \& Meszéna, G. (2006), 'Limiting similarity revisited', Oikos 112(3), 612-619.

Tilman, D. (1982), Resource Competition and Community Structure, Princeton University Press, Princeton, New Jersey. USA.

Vincent, T. L. \& Brown, J. S. (2005), Evolutionary Game Theory, Natural Selection, and Darwinian Dynamics, Cambridge University Press, Cambridge.

Vincent, T. L., Cohen, Y. \& Brown, J. S. (1993), 'Evolution via strategy dynamics', Theoretical Population Biology 44, 149-176.

Volterra, V. (1926), 'Fluctuations in the abundance of a species considered mathematically', Nature 118, 558-560.

Volterra, V. (1928), 'Variations and fluctuations of the number of individuals in animal species living together', J. Cons. Int. Explor. Mer 3(1), 3-51. 
839

840

844

\section{Appendix A: The existence of a symmetric two-species equilibrium for (9)-(12)}

From the main text, when competition between strategies $v$ and $u_{i}$ is modelled by our standard term $a\left(v, u_{i}\right)=\exp \left[-\frac{\left(v-u_{i}\right)^{2}}{2 \sigma_{a}^{2}}\right]$, the symmetric equilibrium corresponds to a solution of the following two equations with $u>0$ and $x>0$.

$$
\begin{aligned}
x e^{-(2 u)^{2} / 2 \sigma_{a}^{2}}+x & =e^{-u^{2} / 2 \sigma_{k}^{2}} \\
\frac{2 u}{\sigma_{a}^{2}} e^{-(2 u)^{2} / 2 \sigma_{a}^{2}} x & =\frac{u}{\sigma_{k}^{2}} e^{-u^{2} / 2 \sigma_{k}^{2}}
\end{aligned}
$$

Since $u \neq 0$, the second equation implies that $x=\frac{\sigma_{a}^{2}}{2 \sigma_{k}^{2}} e^{-u^{2} / 2 \sigma_{k}^{2}} e^{(2 u)^{2} / 2 \sigma_{a}^{2}}$. Substituting this into the first equation and canceling $e^{-u^{2} / 2 \sigma_{k}^{2}}$ yields:

$$
\begin{aligned}
\frac{\sigma_{a}^{2}}{2 \sigma_{k}^{2}} e^{(2 u)^{2} / 2 \sigma_{a}^{2}} e^{-(2 u)^{2} / 2 \sigma_{a}^{2}}+\frac{\sigma_{a}^{2}}{2 \sigma_{k}^{2}} e^{(2 u)^{2} / 2 \sigma_{a}^{2}} & =1 \\
e^{2 u^{2} / \sigma_{a}^{2}} & =\frac{2 \sigma_{k}^{2}}{\sigma_{a}^{2}}\left(1-\frac{\sigma_{a}^{2}}{2 \sigma_{k}^{2}}\right) \\
\frac{2 u^{2}}{\sigma_{a}^{2}} & =\ln \left(\frac{2 \sigma_{k}^{2}}{\sigma_{a}^{2}}-1\right) \\
u^{2} & =\frac{\sigma_{a}^{2}}{2} \ln \left(\frac{2 \sigma_{k}^{2}-\sigma_{a}^{2}}{\sigma_{a}^{2}}\right)
\end{aligned}
$$

Thus, 


$$
\begin{aligned}
x & =\frac{\sigma_{a}^{2}}{2 \sigma_{k}^{2}} e^{-u^{2} / 2 \sigma_{k}^{2}} e^{(2 u)^{2} / 2 \sigma_{a}^{2}} \\
& =\frac{\sigma_{a}^{2}}{2 \sigma_{k}^{2}} \exp \left[\left(\frac{\sigma_{a}^{2}}{2} \ln \left(\frac{2 \sigma_{k}^{2}-\sigma_{a}^{2}}{\sigma_{a}^{2}}\right)\right)\left(\frac{2}{\sigma_{a}^{2}}-\frac{1}{2 \sigma_{k}^{2}}\right)\right] \\
& =\frac{\sigma_{a}^{2}}{2 \sigma_{k}^{2}} \exp \left[\left(1-\frac{\sigma_{a}^{2}}{4 \sigma_{k}^{2}}\right) \ln \left(\frac{2 \sigma_{k}^{2}-\sigma_{a}^{2}}{\sigma_{a}^{2}}\right)\right] \\
& =\frac{\sigma_{a}^{2}}{2 \sigma_{k}^{2}}\left(\frac{2 \sigma_{k}^{2}-\sigma_{a}^{2}}{\sigma_{a}^{2}}\right)^{1-\frac{\sigma_{a}^{2}}{4 \sigma_{k}^{2}}} .
\end{aligned}
$$

848

\section{Appendix B: Uniqueness of the equilibrium (13), (14)}

Now suppose that there is a non-symmetric two-species equilibrium. That is, there are $u_{1} \neq u_{2}$ and positive $x_{1}, x_{2}$ such that

$$
\begin{aligned}
x_{1}+x_{2} e^{-\left(u_{1}-u_{2}\right)^{2} / 2 \sigma_{a}^{2}} & =e^{-u_{1}^{2} / 2 \sigma_{k}^{2}} \\
x_{1} e^{-\left(u_{1}-u_{2}\right)^{2} / 2 \sigma_{a}^{2}}+x_{2} & =e^{-u_{2}^{2} / 2 \sigma_{k}^{2}} \\
\frac{u_{1}-u_{2}}{\sigma_{a}^{2}} e^{-\left(u_{1}-u_{2}\right)^{2} / 2 \sigma_{a}^{2}} x_{2} & =\frac{u_{1}}{\sigma_{k}^{2}} e^{-u_{1}^{2} / 2 \sigma_{k}^{2}} \\
\frac{u_{2}-u_{1}}{\sigma_{a}^{2}} e^{-\left(u_{1}-u_{2}\right)^{2} / 2 \sigma_{a}^{2}} x_{1} & =\frac{u_{2}}{\sigma_{k}^{2}} e^{-u_{2}^{2} / 2 \sigma_{k}^{2}}
\end{aligned}
$$

From the last two equations, $u_{1}$ and $u_{2}$ are non-zero and have opposite signs. Without loss of generality, assume that $u_{2}=-\theta u_{1}$ where $\theta>1$. With $u \equiv u_{1},(18)$ and (19) imply 


$$
\begin{aligned}
x_{1} & =\frac{\theta u}{(\theta+1) u} \frac{\sigma_{a}^{2}}{\sigma_{k}^{2}} \frac{e^{-(\theta u)^{2} / 2 \sigma_{k}^{2}}}{e^{-((\theta+1) u)^{2} / 2 \sigma_{a}^{2}}} \\
& =\frac{\theta}{(\theta+1)} \frac{\sigma_{a}^{2}}{\sigma_{k}^{2}} \frac{e^{-(\theta u)^{2} / 2 \sigma_{k}^{2}}}{e^{-((\theta+1) u)^{2} / 2 \sigma_{a}^{2}}} \\
x_{2} & =\frac{1}{(\theta+1)} \frac{\sigma_{a}^{2}}{\sigma_{k}^{2}} \frac{e^{-u^{2} / 2 \sigma_{k}^{2}}}{e^{-((\theta+1) u)^{2} / 2 \sigma_{a}^{2}}} .
\end{aligned}
$$

Substituting these values into (16) yields:

$$
\begin{aligned}
& \frac{\sigma_{a}^{2}}{\sigma_{k}^{2}}\left[\frac{\theta}{(\theta+1)} \frac{e^{-(\theta u)^{2} / 2 \sigma_{k}^{2}}}{e^{-((\theta+1) u)^{2} / 2 \sigma_{a}^{2}}}+\frac{1}{(\theta+1)} e^{-u^{2} / 2 \sigma_{k}^{2}}\right]=e^{-u^{2} / 2 \sigma_{k}^{2}} \\
& \frac{\theta}{(\theta+1)} e^{\left(1-\theta^{2}\right) u^{2} / 2 \sigma_{k}^{2}+(\theta+1)^{2} u^{2} / 2 \sigma_{a}^{2}}+\frac{1}{(\theta+1)}=\frac{\sigma_{k}^{2}}{\sigma_{a}^{2}} \\
& \frac{\left(1-\theta^{2}\right) u^{2}}{2 \sigma_{k}^{2}}+\frac{(\theta+1)^{2} u^{2}}{2 \sigma_{a}^{2}}=\ln \left(\frac{\theta+1}{\theta} \frac{\sigma_{k}^{2}}{\sigma_{a}^{2}}-\frac{1}{\theta}\right) \\
& u^{2}=\frac{1}{\frac{1-\theta^{2}}{2 \sigma_{k}^{2}}+\frac{(\theta+1)^{2}}{2 \sigma_{a}^{2}}} \ln \left(\frac{\theta+1}{\theta} \frac{\sigma_{k}^{2}}{\sigma_{a}^{2}}-\frac{1}{\theta}\right)
\end{aligned}
$$

Similarly, from (17), we obtain

$$
u^{2}=\frac{1}{\frac{\theta^{2}-1}{2 \sigma_{k}^{2}}+\frac{(\theta+1)^{2}}{2 \sigma_{a}^{2}}} \ln \left((\theta+1) \frac{\sigma_{k}^{2}}{\sigma_{a}^{2}}-\theta\right) .
$$

Equating these two expressions for $u^{2}$ and rearranging terms produces the following equations where $R \equiv \frac{\sigma_{k}^{2}}{\sigma_{a}^{2}}$ : 


$$
\begin{aligned}
0= & \left(\theta^{2}-1\right)\left[\ln \left(\frac{\theta+1}{\theta} \frac{\sigma_{k}^{2}}{\sigma_{a}^{2}}-\frac{1}{\theta}\right)+\ln \left((\theta+1) \frac{\sigma_{k}^{2}}{\sigma_{a}^{2}}-\theta\right)\right] \\
& +\frac{\sigma_{k}^{2}}{\sigma_{a}^{2}}(\theta+1)^{2}\left[\ln \left(\frac{\theta+1}{\theta} \frac{\sigma_{k}^{2}}{\sigma_{a}^{2}}-\frac{1}{\theta}\right)-\ln \left((\theta+1) \frac{\sigma_{k}^{2}}{\sigma_{a}^{2}}-\theta\right)\right] \\
0= & \left(\theta^{2}-1\right) \ln \left[\left(R+\frac{1}{\theta}(R-1)\right)(R+\theta(R-1))\right]+R(\theta+1)^{2} \ln \left[\frac{R+\frac{1}{\theta}(R-1)}{R+\theta(R-1)}\right]
\end{aligned}
$$

Fix $\theta>1$ and consider the function $f(R) \equiv\left(\theta^{2}-1\right) \ln \left[\left(R+\frac{1}{\theta}(R-1)\right)(R+\theta(R-1))\right]+$ $R(\theta+1)^{2} \ln \left[\frac{R+\frac{1}{\theta}(R-1)}{R+\theta(R-1)}\right]$. Since $\sigma_{k}^{2}>\sigma_{a}^{2}$, we want to show that $f(R)$ is never 0 for $R>1$. This contradiction will complete the proof that there is no non symmetric equilibrium. To see this, we need the first and second derivatives of $f(R)$ :

$$
\begin{aligned}
f^{\prime}(R)= & \left(\theta^{2}-1\right)\left[\frac{\theta+1}{\theta R+R-1}+\frac{\theta+1}{\theta R+R-\theta}\right]+R(\theta+1)^{2}\left[\frac{\theta+1}{\theta R+R-1}-\frac{\theta+1}{\theta R+R-\theta}\right] \\
& +(\theta+1)^{2} \ln \left[\frac{\theta R+(R-1)}{R+\theta(R-1)} \frac{1}{\theta}\right] \\
= & \left(\theta^{2}-1\right)(\theta+1)^{2} \frac{R-1}{(\theta R+R-1)(\theta R+R-\theta)}+(\theta+1)^{2} \ln \left[\frac{\theta R+(R-1)}{R+\theta(R-1)} \frac{1}{\theta}\right] \\
f^{\prime \prime}(R)= & -\left(\theta^{2}-1\right)(\theta+1)^{2} \frac{(R-1)(\theta+1)((\theta R+R-1)+(\theta R+R-\theta))}{(\theta R+R-1)^{2}(\theta R+R-\theta)^{2}} \\
& +\frac{\left(\theta^{2}-1\right)(\theta+1)^{2}}{(\theta R+R-1)(\theta R+R-\theta)}-(\theta+1)^{2} \frac{\left(\theta^{2}-1\right)}{(\theta R+R-1)(\theta R+R-\theta)} \\
= & -\frac{\left(\theta^{2}-1\right)(\theta+1)^{3}(R-1)(2 R-1)}{(\theta R+R-1)^{2}(\theta R+R-\theta)^{2}}
\end{aligned}
$$

Thus $f(1) \equiv\left(\theta^{2}-1\right) \ln 1+(\theta+1)^{2} \ln 1=0, f^{\prime}(1)=\left(\theta^{2}-1\right)(\theta+1)^{2} \frac{0}{\theta}+(\theta+1)^{2} \ln 1=0$ and $f^{\prime \prime}(R)<0$ for all $R>\frac{1}{2}$. In particular, by concavity, $f(R)<0$ for $R>1$ and this completes the proof. 


\section{Appendix C: Speciation at the symmetric equilibrium}

\section{$(13),(14)$}

Our final claim for this model is that, for the two-species symmetric equilibrium, the adaptive landscape is at a minimum value of 0 exactly at $u_{1}^{*}=u$ and $u_{2}^{*}=-u$. We want to show that $G\left(v,\left(u_{1}^{*}, u_{2}^{*}\right),\left(x_{1}^{*}, x_{2}^{*}\right)\right)$ is at a minimum as a function of $v$ at $u$ and $-u$. That is

$$
\left.\frac{\partial^{2} G(v, \mathbf{u}, \mathbf{x})}{\partial v^{2}}\right|_{v= \pm u}>0
$$

Taking the second derivative of the fitness function

$$
G(v, \mathbf{u}, \mathbf{x})=\frac{r}{K(v)}\left[K(v)-\sum_{j=1}^{n} a\left(v, u_{j}\right) x_{j}\right]
$$

and simplifying by using the eco- and evo-equilibrium conditions (6) and (8) we obtain

$$
\frac{\partial^{2} G(v, \mathbf{u}, \mathbf{x})}{\partial v^{2}}=-\frac{r}{K(v)}\left[\sum_{j=1}^{n} \frac{\partial^{2} a\left(v, u_{j}\right)}{\partial v^{2}} x_{j}-K^{\prime}(v)\right]
$$

Thus for $G(v, \mathbf{u}, \mathbf{x})$ to be at local minimum for for each $v=u_{i}, i=1, \cdots, n$

$$
\left.\left[\sum_{j=1}^{n} \frac{\partial^{2} a\left(v, u_{j}\right)}{\partial v^{2}} x_{j}-K^{\prime}(v)\right]\right|_{v=u_{i}}<0
$$

Furthermore, we assume that competition is most intense between individuals using the same strategy. Like competes most with like. Hence, we let the competition coefficient, $a\left(v, u_{j}\right)$, be a Gaussian relationship based on the difference between the focal individual's strategy and that of the competitor. The function reaches a maximum of 1 when $v=u_{i}$ and declines as the strategies of the competitors diverge. This yields the following: 


$$
K^{\prime}(v)=\left[\left(\frac{v}{\sigma_{k}^{2}}\right)^{2}-\frac{1}{\sigma_{k}^{2}}\right] K(v) \text { and } \frac{\partial^{2} a\left(v, u_{j}\right)}{\partial v^{2}}=\left[\left(\frac{v-u_{j}}{\sigma_{a}^{2}}\right)^{2}-\frac{1}{\sigma_{a}^{2}}\right] a\left(v, u_{j}\right)
$$

Substituting expressions for these derivatives into the LHS of the minima condition we have two equivalent expressions

$$
\begin{gathered}
\frac{1}{\sigma_{a}^{2}} \sum_{j=1}^{n} u_{j} x_{j} \frac{\partial \alpha\left(v, u_{j}\right)}{\partial v}+\frac{v^{2}}{\sigma_{k}^{2}}\left[\frac{1}{\sigma_{a}^{2}}-\frac{1}{\sigma_{k}^{2}}\right] K(v)-\left[\frac{1}{\sigma_{a}^{2}}-\frac{1}{\sigma_{k}^{2}}\right] K(v) \\
\sum_{j=1}^{n} \frac{x_{j}}{\alpha\left(v, u_{j}\right)}\left(\frac{\partial \alpha\left(v, u_{j}\right)}{\partial v}\right)^{2}-\frac{v^{2}}{\sigma_{k}^{4}} K(v)-\left[\frac{1}{\sigma_{a}^{2}}-\frac{1}{\sigma_{k}^{2}}\right] K(v) .
\end{gathered}
$$

Using the last expression for $n=2$ species with $u_{1}=u$ and $u_{2}=-u$ (symmetric solution) with $v=u_{1}$ or $v=u_{2}$, expanding and simplifying only the summation we get

$$
\frac{2 u^{2}}{\sigma_{a}^{2} \sigma_{k}^{2}} K(u)
$$

Substituting this back into the whole expression and ignoring $K(u)$ we obtain

$$
\frac{2 \sigma_{k}^{2}-\sigma_{a}^{2}}{\left(\sigma_{k}^{2}\right)^{2}} \frac{1}{2} \ln \left(\frac{2 \sigma_{k}^{2}-\sigma_{a}^{2}}{\sigma_{a}^{2}}\right)-\frac{\sigma_{k}^{2}-\sigma_{a}^{2}}{\sigma_{a}^{2} \sigma_{k}^{2}}
$$

or

$$
\frac{1}{\sigma_{k}^{2}}\left\{\frac{2 \sigma_{k}^{2}-\sigma_{a}^{2}}{\sigma_{k}^{2}} \frac{1}{2} \ln \left(\frac{2 \sigma_{k}^{2}-\sigma_{a}^{2}}{\sigma_{a}^{2}}\right)-\frac{\sigma_{k}^{2}-\sigma_{a}^{2}}{\sigma_{a}^{2}}\right\}
$$

Now fix $\sigma_{a}^{2}$ and let $\sigma_{k}^{2}=\phi \sigma_{a}^{2}$. The above expression can be written as

$$
\frac{1}{2 \phi \sigma_{a}^{2}}\left[(2 \phi-1) \ln (2 \phi-1)-2\left(\phi^{2}-\phi\right)\right]
$$


Since $\frac{1}{2 \phi \sigma_{a}^{2}}>0$ the sign of the expression is determined by $f(\phi)=(2 \phi-1) \ln (2 \phi-1)-$ $2\left(\phi^{2}-\phi\right)$. First note that $f(1)=0$. In addition

$$
f^{\prime}(\phi)=2[\ln (2 \phi-1)-(2 \phi-1)+1] \text { and } f^{\prime \prime}(\phi)=4\left[\frac{2-2 \phi}{2 \phi-1}\right]
$$

For $\phi>1, f^{\prime \prime}(\phi)<0$ and $f(\phi)$ is concave down. Since $f(1)=0$ it follows that $f(\phi)<0$ for $\phi>1$. (Alternatively, note that for $\phi>1, f^{\prime \prime}(\phi)<0$ and $f^{\prime}(\phi)$ is decreasing. Since $f^{\prime}(1)=0$, it follows that $f^{\prime}(\phi)<0$. Thus $f(\phi)$ is decreasing and with $f(1)=0$ we conclude that $f(\phi)<0$ for $\phi>1$.) This establishes the proof for the minima.

Thus, the equilibrium is at a minimum of the two species G-function.

\section{Appendix D: Details of the simulations}

Using the software package R (v. 3.1.1) (R Core Team 2013), we developed a series of numerical programs to simulate the ecological and evolutionary dynamics of a descretized version (Vincent and Brown 2005, Eqn. (4.5.2) and (5.9.2)) of the model

$$
\frac{\partial x_{i}}{\partial t}=x_{i} G(v, \mathbf{u}, \mathbf{x}) \text { when } v=u_{i} \quad i=1, \cdots, n \quad \text { (ecological dynamics) }
$$

and

$$
\frac{\partial u_{i}}{\partial t}=k \frac{\partial G}{\partial v} \text { when } v=u_{i} \quad i=1, \cdots, n \quad \text { (evolutionary dynamics) }
$$

The community began with a single species, with initial conditions of $u_{1}=0.1$ and $x_{1}=$ 1. The simulation would automatically run for as many generations as necessary to reach a convergent stable equilibrium. Using the sign of the second derivative, a subroutine would then evaluate each species' strategy for whether it was as a minima or maxima on the adaptive landscape. If all species' strategies were at maxima on the adaptive landscape, 
then the simulation would stop (E.g. figure $2 \mathrm{~A}-\mathrm{C}$ ). On the other hand, if a strategy was at a minimum on the adaptive landscape then the simulaition treated it as a bifurcation point where a mutation could lead to speciation (e.g., figure 2 D-F). At such a bifurcation point, the program automatically added a second species with $u_{1}=u^{*}, u_{2}=u^{*}-\delta, x_{1}=x^{*}$ and $x_{2}=1$, where $\delta=0.1$, and the ecological and evolutionary dynamics continued from where they left off prior to speciation and again run to equilibrium (e.g. figure 3). Every time the community evolved to a convergent stable equilibrium, species at a convergent stable minimum were allowed to speciate by adding a new species adjacent to each minimum with a strategy value of $u_{i+1}=u_{i}^{*}-\delta$ and a population size of $x_{i+1}=1$. However, the subprogram that checked whether the strategies are at a minimum on the landscape takes a great deal of computational time. For example, after running the program for 150 hours on a 57 computer cluster, the simulation had run for $\sim 20$ million generations, and still had not reached equilibrium for 64 species. The simulation gets very close to the equilibrium relatively quickly, but then it would take a very long time to get all the way to equilibrium. This can already be seen to some extent in figure $3 \mathrm{~A}$, where the strategy values were identical to 4 decimal places for thousands of generations before equilibrium was fully reached. Since competition coefficients must be calculated among all species within the community, this version of our program would become even more numerically intensive as diversity increased past 64 species. In many cases adding the new species at a population size (this can also be thought of as density) of $x_{2}=1$ can be a sizable fraction of the original species' populaiton size, $x_{1}$. For this model, this is not an issue because all convergent stable points and their associated strategy values and population sizes are global. We verified this by running several of the simiulations with a smaller initial density for the new species $\left(x_{2}=0.001\right)$. All results remained the same.

With $\sigma_{k}^{2}>\sigma_{a}^{2}$, our experience with the simulation up to 64 species showed that the automatic speciation algorithm produced a convergent stable minimum (i.e. before speciation, 
the dynamics reached an equilibrium where all species were at a minimum of the adaptive landscape). Specifically, this led to a doubling of diversity at each equilibrium point. Thus, to speed up our simulations, we wrote a second program that did not check whether all the species were at a minimum on the landscape, but instead just doubled species once the equilibrium point was such that strategy and population values satisfied $G(v, \mathbf{u}, \mathbf{x}) \leq 10^{-7}$ and $\frac{\partial G}{\partial v} \leq 10^{-7}$, at which point all species would speciate as described above, and the dynamics would continue from that point with twice as many species. This species doubling shortcut made the simulations numerically tractable and was allowed to continue according to these simulation rules, and following the Darwinian dynamics defined by the $\mathrm{L}-\mathrm{V}$ game for 3.51 million generations, which produced 1024 species. A subset of the complete ecological and evolutionary dynamics up to 16 species are shown in figure 4 . These 16 species dynamics are produced from the same parameters as figure 2 D-F and figure 3 except speciation was permitted to continue to $n=16$.

\section{Appendix E: Nash equilibrium with full support for ba-}

\section{sic LV model}

Recall from the main text that

$$
G(v, u, x)=\frac{r}{K(v)}\left[K(v)-\sum_{j=1}^{n} a\left(v, u_{j}\right) x_{j}\right]
$$

where the carrying capacity and interaction term have the forms

$$
K(v)=K_{m} \exp \left[-\frac{v^{2}}{2 \sigma_{k}^{2}}\right] ; \quad a(v, u)=\exp \left[-\frac{(v-u)^{2}}{2 \sigma_{a}^{2}}\right] .
$$

If $\sigma_{k}^{2}<\sigma_{a}^{2}$, then there is a CSS for the monomorphic population with strategy $u=0$. 
Assume that $\sigma_{k}^{2}>\sigma_{a}^{2}$. For the top-down approach, we try a normal distribution $P^{*}(u)=$ $\frac{\sqrt{m}}{\sqrt{2 \pi}} e^{-m u^{2} / 2}$ that is adjusted to a total population size of $N^{*}$. Since

$$
G\left(v, u, N^{*}\right)=r\left[1-\frac{N^{*} \exp \left(-(v-u)^{2} / 2 \sigma_{a}^{2}\right)}{K \exp \left(-v^{2} / 2 \sigma_{k}^{2}\right)}\right],
$$

we have that the payoff $\pi\left(v, P^{*}, N^{*}\right)$ to $v$ when the population is in the state $\left(P^{*}, N^{*}\right)$ is

$$
\begin{aligned}
\pi\left(v, P^{*}, N^{*}\right) & =\int_{\mathbf{R}} \pi\left(v, u, N^{*}\right) P^{*}(u) d u \\
& =r \int_{-\infty}^{\infty}\left(1-\frac{N^{*} \exp \left(-(v-u)^{2} / 2 \sigma_{a}^{2}\right.}{K \exp \left(-v^{2} / 2 \sigma_{k}^{2}\right)}\right) \frac{\sqrt{m}}{\sqrt{2 \pi}} e^{-m u^{2} / 2} d u
\end{aligned}
$$

Completing the square in $v$ for the exp exponent, we find that $\pi\left(v, P^{*}, N^{*}\right)$ is independent of $v$ if and only if

$$
m=2\left[\frac{1}{\left(2 \sigma_{a}^{2}\right)^{2}} \frac{1}{\frac{1}{2 \sigma_{a}^{2}}-\frac{1}{2 \sigma_{k}^{2}}}-\frac{1}{2 \sigma_{a}^{2}}\right] .
$$

If we write $P^{*}(u)=\frac{1}{\sqrt{2 \pi} M} e^{-u^{2} / 2 M^{2}}$ instead (i.e. if we replace $m$ by $\frac{1}{M^{2}}$ ), we find that

$$
M^{2}=\sigma_{k}^{2}-\sigma_{k}^{2}
$$

That is, $P^{*}(u)$ is given by Eqn 15 .

\section{Appendix F: The symmetric equilibrium under baseline competition}

We also have an expression for the two-species symmetric equilibrium when the competition term between $v$ and $u_{i}$ has the form 


$$
p+(1-p) a\left(v, u_{i}\right)=p+(1-p) \exp \left[-\frac{\left(v-u_{i}\right)^{2}}{2 \sigma_{a}^{2}}\right] .
$$

The system of two equations to solve for $u>0$ and $x>0$ are now

$$
\begin{aligned}
x\left(p+(1-p) e^{-(2 u)^{2} / 2 \sigma_{a}^{2}}\right)+x & =e^{-u^{2} / 2 \sigma_{k}^{2}} \\
\frac{2 u}{\sigma_{a}^{2}}(1-p) e^{-(2 u)^{2} / 2 \sigma_{a}^{2}} x & =\frac{u}{\sigma_{k}^{2}} e^{-u^{2} / 2 \sigma_{k}^{2}} .
\end{aligned}
$$

Since $u \neq 0$, the second equation implies that $x=\frac{1}{1-p} \frac{\sigma_{a}^{2}}{2 \sigma_{k}^{2}} e^{-u^{2} / 2 \sigma_{k}^{2}} e^{2 u^{2} / \sigma_{a}^{2}}$. Substituting this into the first equation and canceling $e^{-u^{2} / 2 \sigma_{k}^{2}}$ yields:

$$
\frac{1}{1-p} \frac{\sigma_{a}^{2}}{2 \sigma_{k}^{2}} e^{2 u^{2} / \sigma_{a}^{2}}\left(1+p+(1-p) e^{-(2 u)^{2} / 2 \sigma_{a}^{2}}\right)=1 .
$$

Thus,

$$
\begin{aligned}
u^{2} & =\frac{\sigma_{a}^{2}}{2} \ln \left(\frac{2 \sigma_{k}^{2}-\sigma_{a}^{2}}{\sigma_{a}^{2}} \frac{1-p}{1+p}\right) \\
x & =\frac{1}{1+p} \frac{\sigma_{a}^{2}}{2 \sigma_{k}^{2}}\left(\frac{2 \sigma_{k}^{2}-\sigma_{a}^{2}}{\sigma_{a}^{2}}\right)^{1-\frac{\sigma_{a}^{2}}{4 \sigma_{k}^{2}}}
\end{aligned}
$$

as claimed in the main text. Since $u^{2}>0$, we must have $\frac{2 \sigma_{k}^{2}-\sigma_{a}^{2}}{\sigma_{a}^{2}} \frac{1-p}{1+p}>1$. Solving for $p$, we have that $p<p_{1}^{*} \equiv 1-\frac{\sigma_{a}^{2}}{\sigma_{k}^{2}}$. That is, the two-species symmetric equilibrium exists if and only if $0 \leq p<1-\frac{\sigma_{a}^{2}}{\sigma_{k}^{2}}$. 


\section{Appendix G: The symmetric equilibrium under trun-}

\section{cated trait space}

In section "The effect of truncated trait space", we replaced the carrying capacity $K(v)=$ $K_{m} e^{-v^{2} / 2 \sigma_{k}^{2}}$ in $(3)$ by

$$
\widehat{K}(v) \equiv\left\{\begin{array}{cl}
K(v)+s\left(K(v)-K_{m}\right) & \text { if } K(v)+s\left(K(v)-K_{m}\right)>0 \\
0 & \text { if } K(v)+s\left(K(v)-K_{m}\right) \leq 0
\end{array} .\right.
$$

Here $s>0$ is a shrinking parameter in that the carrying capacity of the model is supported on the interval in trait space $v$ where $K(v)+s\left(K(v)-K_{m}\right)>0$. Since $K(v)+s\left(K(v)-K_{m}\right)=$ $K_{m}\left((1+s) e^{-v^{2} / 2 \sigma_{k}^{2}}-s\right)$, we find that $\widehat{K}(v)>0$ when $-v^{2} / 2 \sigma_{k}^{2}>\ln \left(\frac{s}{1+s}\right)$. Thus, the support of $\widehat{K}$ (i.e. the closure of the set where $\widehat{K}$ is non-zero) is the finite interval in trait space given by $v^{2}<2 \sigma_{k}^{2} \ln \left(\frac{1+s}{s}\right)$ (i.e. $v \in\left[v_{-}, v_{+}\right]$where $\left.v_{ \pm} \equiv \pm \sqrt{2} \sigma_{k} \sqrt{\ln \left(\frac{1+s}{s}\right)}\right)$.

Since $\widehat{K}(v)$ is 0 for some values of $v$, the G-function given by (1) is undefined for these $v$. To maintain the development of the eco-evolutionary dynamics (4) and (5) in section "An Eco-Evolutionary Lotka-Volterra Model", we formally set $G(v, \mathbf{u}, \mathbf{x}) \equiv-\infty$ whenever $\widehat{K}(v)=0$. This agrees with the limiting values at $v_{ \pm}$in that $\lim _{v \rightarrow v_{+}^{-}} G(v, \mathbf{u}, \mathbf{x})=G\left(v_{+}, \mathbf{u}, \mathbf{x}\right)$ and $\lim _{v \rightarrow v_{-}^{+}} G(v, \mathbf{u}, \mathbf{x})=G\left(v_{-}, \mathbf{u}, \mathbf{x}\right)$. It then makes sense to take $x_{i}=0$ for any $u_{i}$ outside the support of $\widehat{K}$ since, from (4), $x_{i}$ would be decreasing infinitely fast to 0 if it were positive. Thus, any equilibrium of the dynamics corresponds to strategies $u_{i}$ in the interior of the support of $\widehat{K}$. Furthermore, from (2) and (23), $\frac{\partial G}{\partial v}<0$ whenever $v=u_{i}$ is in this interval close to $v_{+}$(in fact, $\lim _{v \rightarrow v_{+}^{-}} \frac{\partial G}{\partial v}=-\infty$ ). Similarly $\lim _{v \rightarrow v_{-}^{+}} \frac{\partial G}{\partial v}=\infty$. Thus, the evolutionary dynamics (5) forces strategies to evolve away from the boundary of the support of $\widehat{K}$ and into its interior.

A two-species symmetric equilibrium from (2) and (23) is given by a $u_{+}=u>0$ and $u_{-}=-u_{+}$in (the interior of) the support of $\widehat{K}$ that satisfies 


$$
\frac{\sigma_{a}^{2}}{2 \sigma_{k}^{2}} e^{2 u^{2} / \sigma_{a}^{2}}=\frac{2 \sigma_{k}^{2}-\sigma_{a}^{2}}{2 \sigma_{k}^{2}}-\frac{s}{1+s} \frac{e^{u^{2} / 2 \sigma_{k}^{2}}}{K_{m}} .
$$

For fixed $s>0$, the LHS of this equation is an increasing function of $u>0$ that increases to $\infty$ and the RHS is a decreasing function of $u>0$ that decreases to $-\infty$. Thus, by evaluating both sides at $u=0$, there is a two-species symmetric equilibrium (which will automatically be unique) if and only if

$$
\begin{aligned}
\frac{\sigma_{a}^{2}}{2 \sigma_{k}^{2}} & <\frac{2 \sigma_{k}^{2}-\sigma_{a}^{2}}{2 \sigma_{k}^{2}}-\frac{s}{1+s} \frac{1}{K_{m}} \\
\frac{s}{1+s} & <K_{m} \frac{\sigma_{k}^{2}-\sigma_{a}^{2}}{\sigma_{k}^{2}} .
\end{aligned}
$$

Since $0 \leq \frac{s}{1+s}<1$ for $s \geq 0$, if $K_{m} \frac{\sigma_{k}^{2}-\sigma_{a}^{2}}{\sigma_{k}^{2}} \geq 1$, then there is a two-species symmetric equilibrium for all $s \geq 0$.

On the other hand, if $K_{m} \frac{\sigma_{k}^{2}-\sigma_{a}^{2}}{\sigma_{k}^{2}}<1$, then there is a two-species symmetric equilibrium if and only if $s<s^{*}$, where

$$
\begin{aligned}
\frac{s^{*}}{1+s^{*}} & =K_{m} \frac{\sigma_{k}^{2}-\sigma_{a}^{2}}{\sigma_{k}^{2}} \\
s^{*} & \equiv \frac{\left(\sigma_{k}^{2}-\sigma_{a}^{2}\right) K_{m}}{\sigma_{k}^{2}+\left(\sigma_{a}^{2}-\sigma_{k}^{2}\right) K_{m}} .
\end{aligned}
$$

Note that $s^{*}>0$ when $K_{m} \frac{\sigma_{k}^{2}-\sigma_{a}^{2}}{\sigma_{k}^{2}}<1$ since $\sigma_{k}^{2}+\left(\sigma_{a}^{2}-\sigma_{k}^{2}\right) K_{m}>0$ and $\sigma_{k}^{2}>\sigma_{a}^{2}$. 

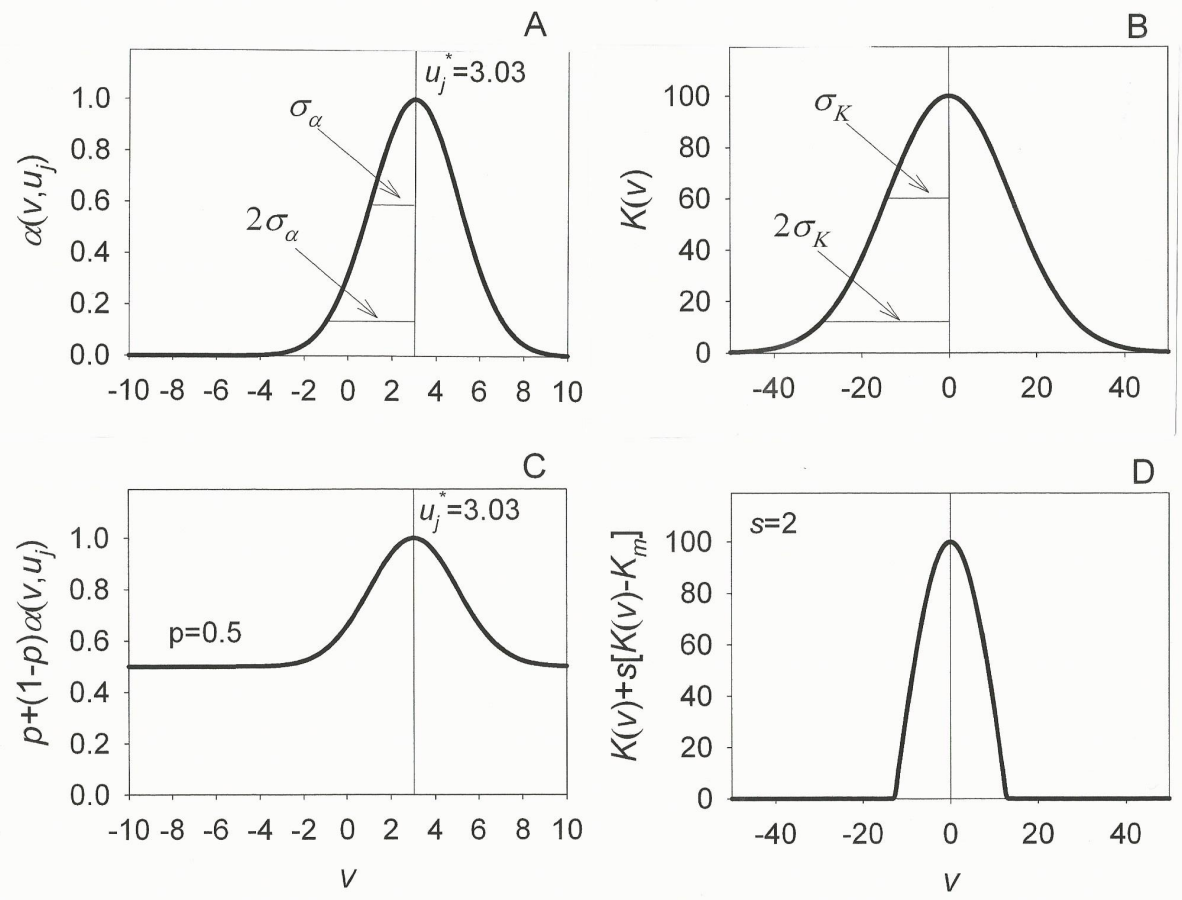

Figure 1: Examples of the effect of strategy $(v)$ and the parameters $\sigma_{k}$ on the competition coefficient and the carrying capacity. (A) The competition coefficient $a\left(v, u_{j}\right)$ for $\sigma_{a}=2$ and $u_{j}=3.0314$ has a maximum at $v=3.0314$. As the focal strategy $(v)$ becomes more different from that of it's competitors $\left(u_{j}\right)$, competition approaches zero causing disruptive selection. (B) Carrying capacity, $K(v)$, for $\sigma_{k}^{2}=200$ is at a maximum when $v=0$ causing stabilizing selection. (C) We can change the baseline level of competition from 0 to $\mathrm{p}$ to examine the effect of slowly removing the disruptive selection caused by competition on adaptive dynamics and niche packing. In this example, the minimum value for the competition coefficient is $p=0.5$. (D) Similarly, we can truncate the distribution for $K(v)$ by dropping it to the $v$-axis with the shrinking parameter $s$, to examine the effect of narrowing the niche width on infinite niche packing. In this example, $s=2$ and $K(v)$ is effectively truncated at $v= \pm 12.73$. 

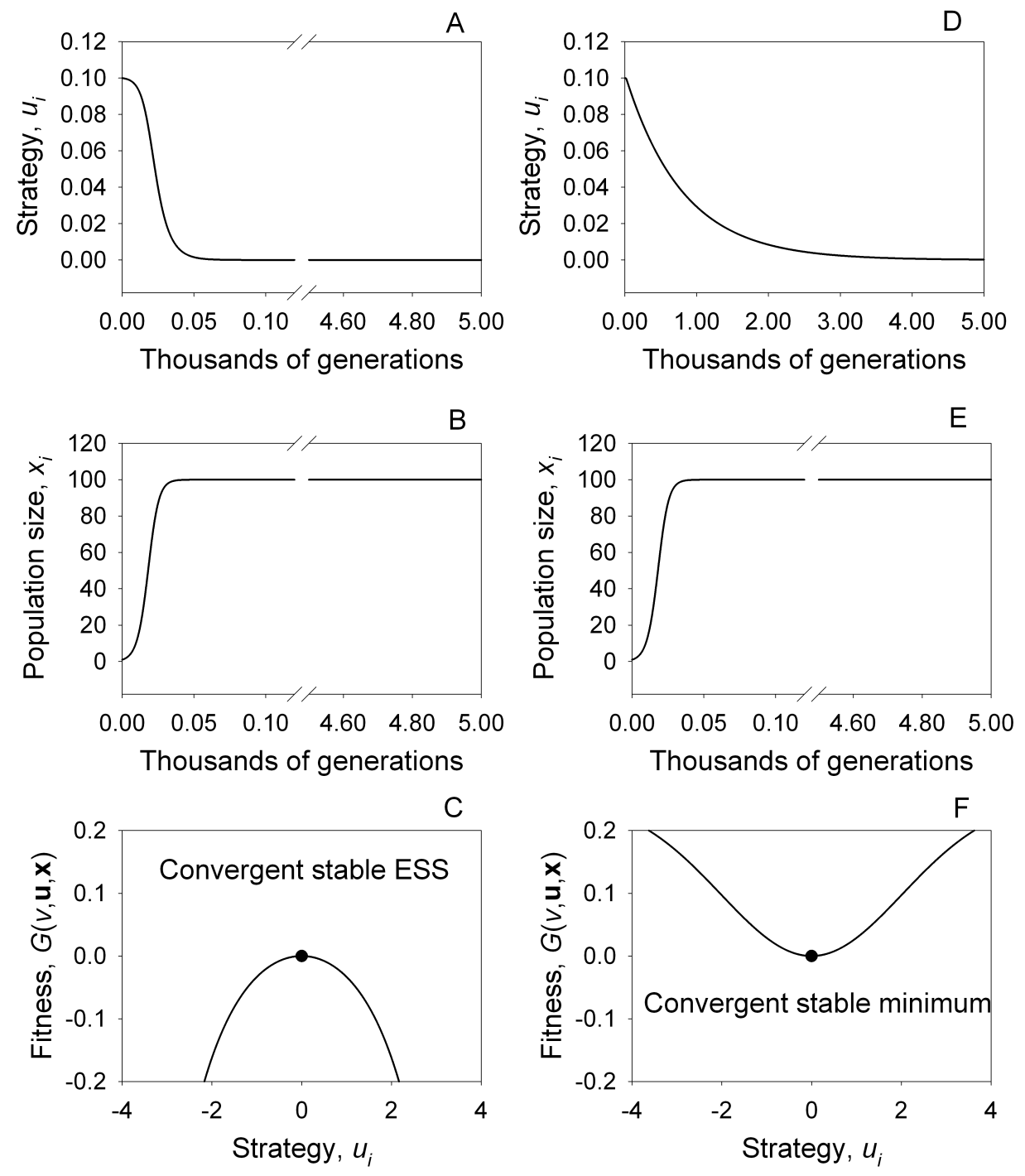

Figure 2: The adaptive dynamics for one species that goes to an ESS (A-C), or for one species that goes to a bifurcation point (D-F). Panels (A, D) show the strategy dynamics, panels $(\mathrm{B}, \mathrm{E})$ show the population dynamics and panels $(\mathrm{C}, \mathrm{F})$ show the final adaptive landscapes. In all panels $r=0.25, K_{m}=100, k=1$. For panels (A-C) the parameters are $\sigma_{k}=\sqrt{2}$ and $\sigma_{a}=2$, for panels (d-f) $\sigma_{k}=\sqrt{200}$ and $\sigma_{a}=2$. 

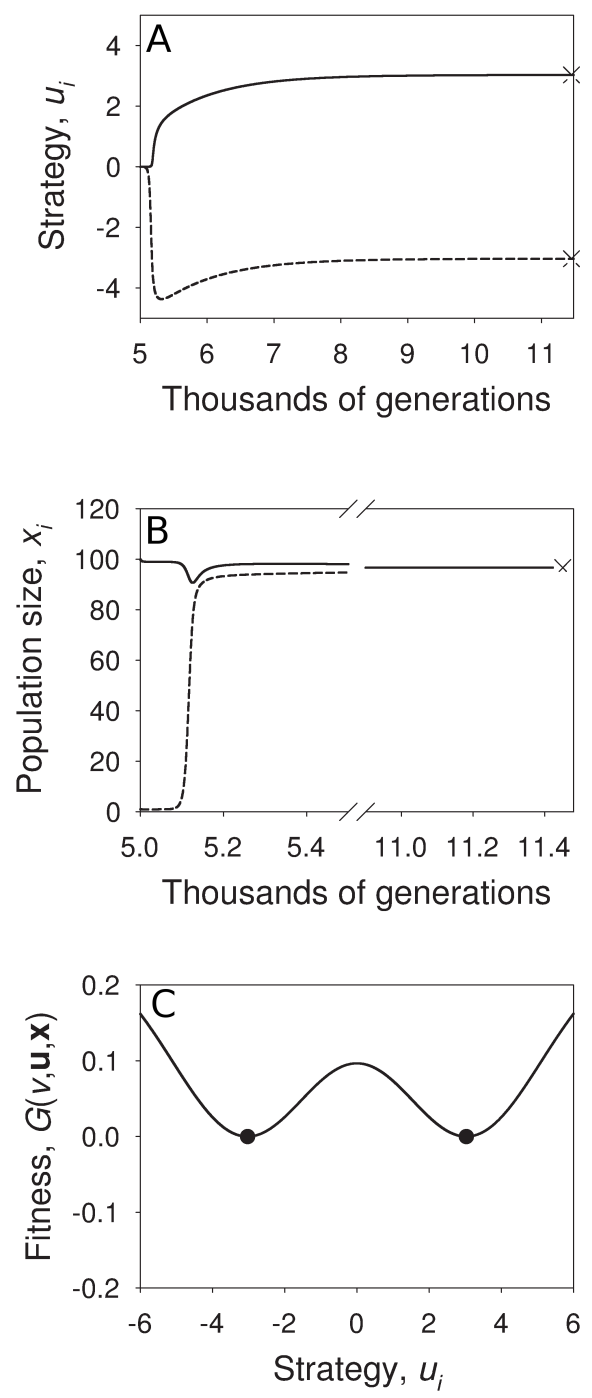

Figure 3: The two species dynamics for parameter values $r=0.25, K_{m}=100, k=1$, $\sigma_{k}=\sqrt{200}$ and $\sigma_{a}=2$. This is a continuation of the dynamics in Figure 1 (D-F) assuming a mutation led to speciation from the convergent stable minimum. The analytic solutions for two species are given by Eqn 5 and Eqn 6, and for these parameters are $u^{*}= \pm 3.031541$ and $x^{*}=96.75135$. These analytic solutions are shown by $X$ in (A) and (B) respectively and match the numerical solutions. Panel $(\mathrm{C})$ shows the final adaptive landscape. 

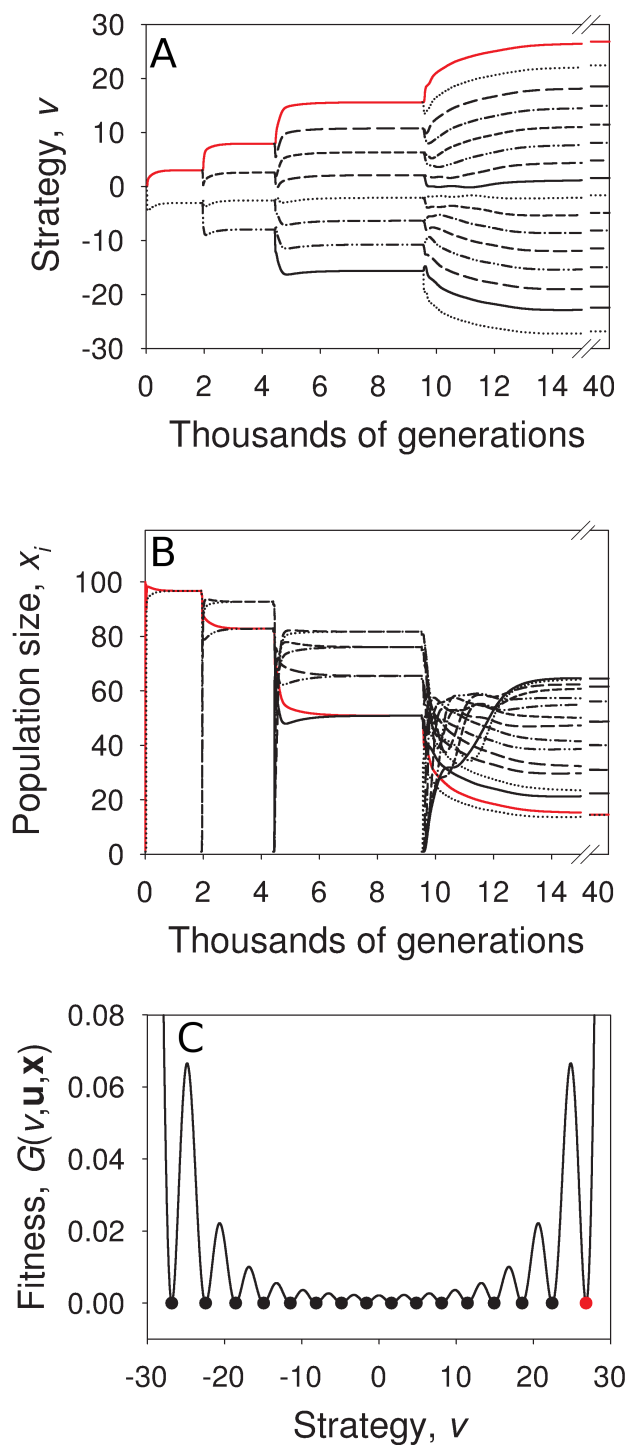

Figure 4: Example of eco-evolutionary dynamics starting from a single common ancestor $\left(u^{*}=0\right)$ and evolving into a community of 16 species. The dynamics of the "original" species are shown in red. The evolutionary process was artificially stopped at 16 species, but we hypothesize that doubling will proceed indefinitely. (A) The phylogeny of strategy dynamics over time, each time an evolutionary minimum is reached, a mutation causes speciation. (B) The population dynamics associated with the phylogeny, each time speciation occurs the new species begins from a population size of 1. (C) The final adaptive landscape for 16 species just before speciation. These are the same dynamics as shown in Fig 2 D-F and Fig 3 , but with speciation at each convergent stable minimum point. Parameters were $r=0.25$, $K_{m}=100, k=1, \sigma_{k}=\sqrt{200}$ and $\sigma_{a}=2$. 

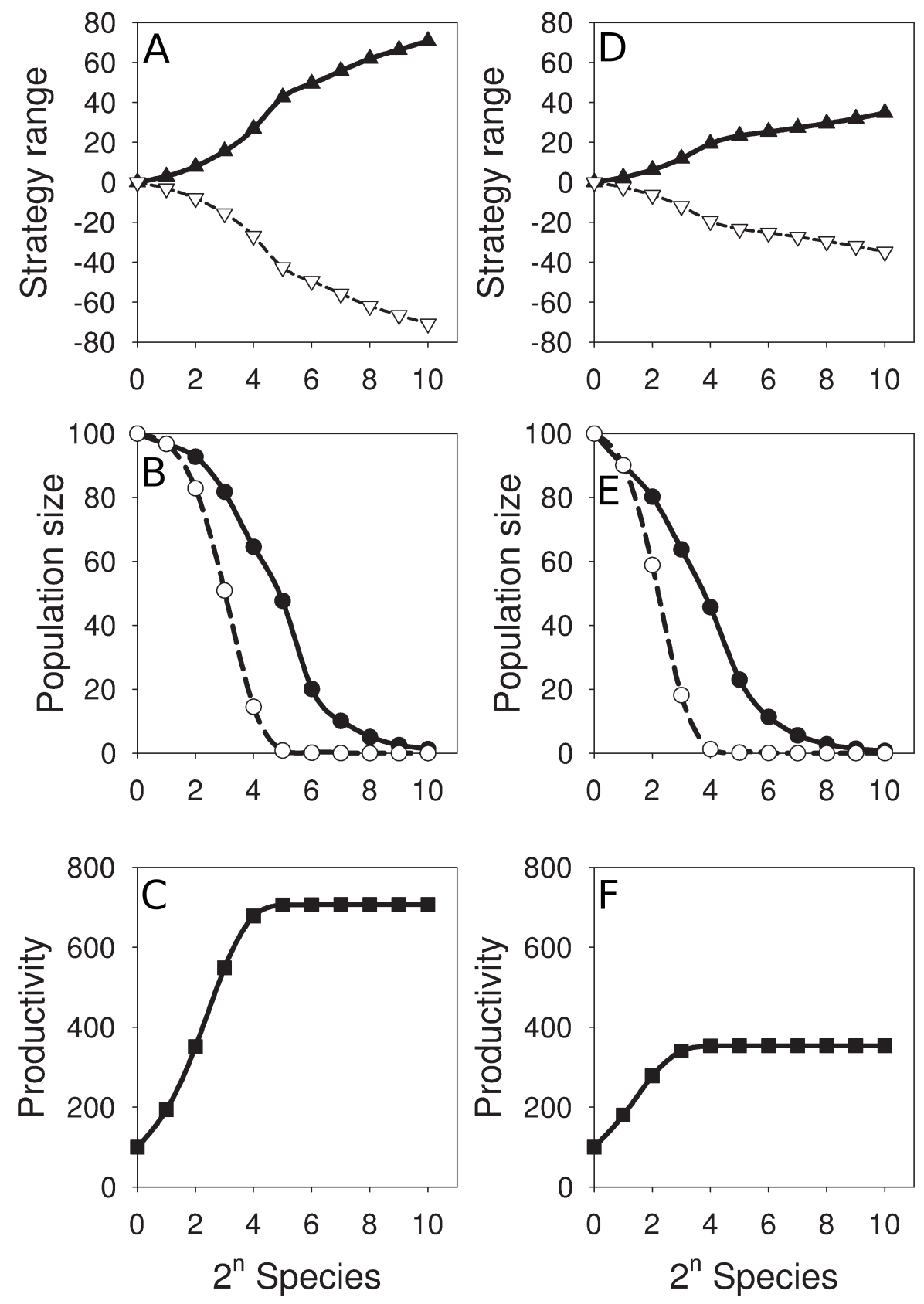

Figure 5: Eco-evolutionary outcomes up to 1024 species showing how increasing niche packing causes the maximum range of strategies to expand (A, D), and the population size of each species to decline (B, E). Productivity, the total population size of all species, increases to a maximum. In all panels $r=0.25, K_{m}=100, k=1, \sigma_{a}=2 . \sigma_{k}^{2}$ was varied, and panels (A)-(C) are for $\sigma_{k}^{2}=50$ and panels (D)-(F) are for $\sigma_{k}^{2}=200$. 

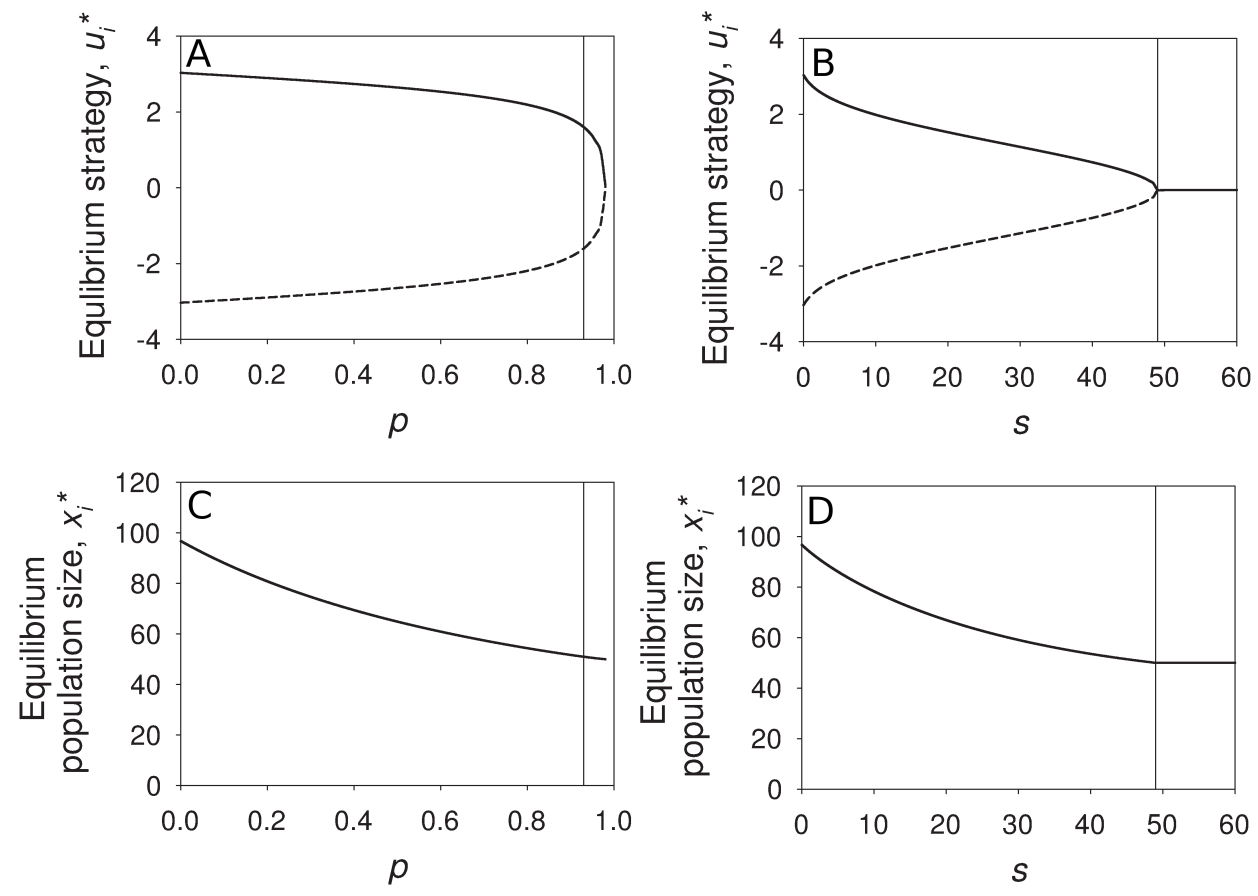

Figure 6: The effect of changing the competition function form $a\left(v, u_{i}\right)$ to $p+(1-p) a\left(v, u_{i}\right)$ where $0 \leq p<1$ models baseline competition on the two species equilibrium strategy (A) and equilibrium population size (B) shown in Fig 3. For $0 \leq p \leq 0.93$ the equilibrium strategy is at a bifurcation point, for $0.93<p \leq 0.98$ the equilibrium strategy is at a maximum, $p=0.93$ is denoted by the vertical line in (A) and (B). Also shown, the effect of truncating the $K$ function by introducing a shrinking parameter s as in equation (15) on the two species equilibrium (C) and equilibrium population size (D). For $0 \leq s \leq 49$ the equilibrium strategy is a at a bifurcation point, for $s>49$ the equilibrium strategy is at a maximum; $s=49$ is denoted by the vertical line in (C) and (D). Parameters were $r=0.25, K_{m}=100, k=1$, $\sigma_{k}=\sqrt{200}$ and $\sigma_{a}=2$. 

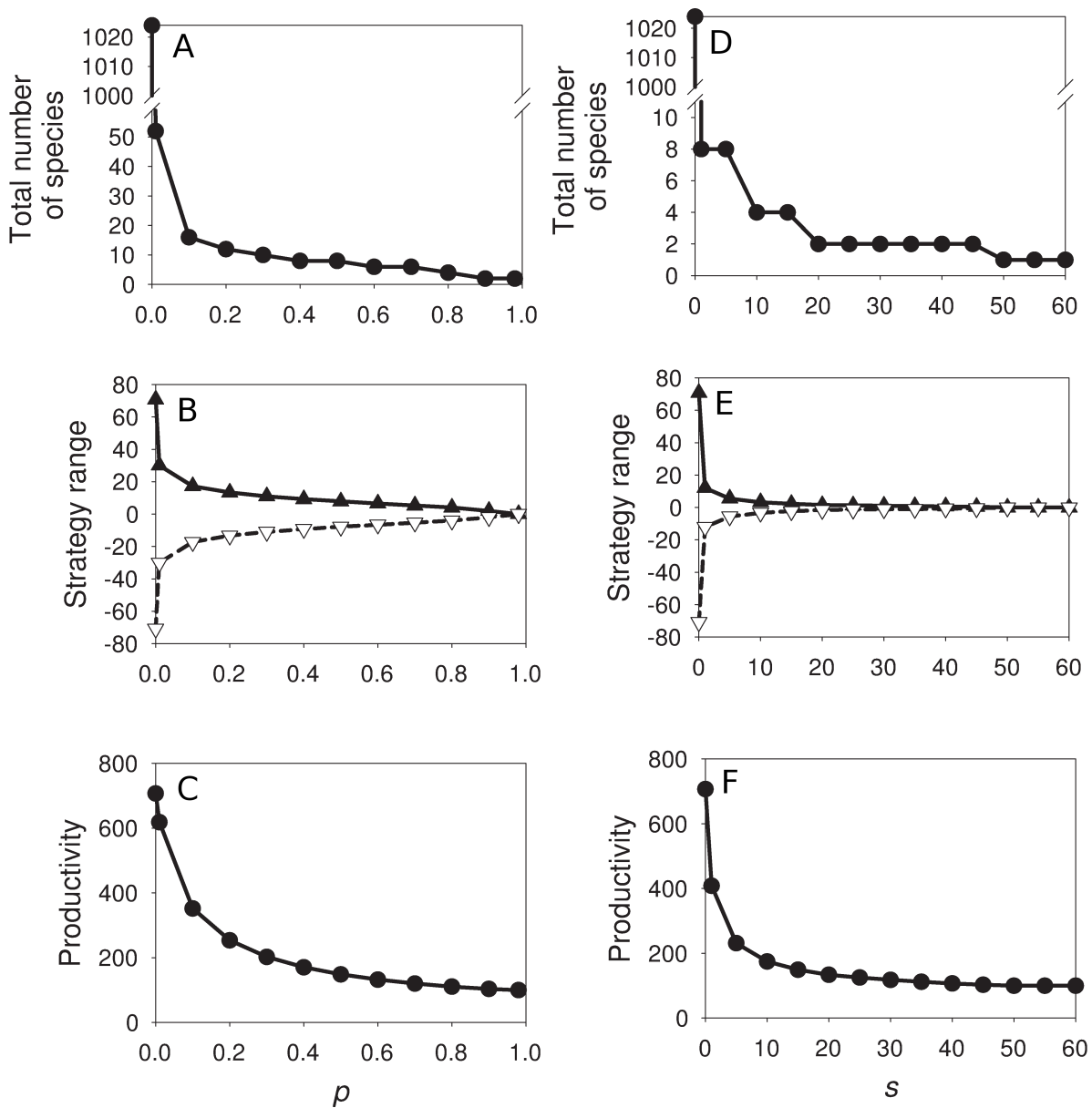

Figure 7: Adjusting the baseline level of competition by introducing $p$ into the Alpha function (A-C), or truncating the carrying capacity by introducing $s$ into the $K$ function (D-F) changes niche packing. Very small levels of baseline competition cause dramatic drops in (A) total species (defined as the number of unique equilibrium strategies), (B) the range of strategies and $(\mathrm{C})$ the total productivity (total population size of all species) of the system. 